\title{
Effectiveness of interventions to prevent falls for people with multiple sclerosis, Parkinson's disease and stroke: an umbrella review
}

Nicola O’Malley ${ }^{1,2^{*}}$, Amanda M. Clifford ${ }^{1,2}$, Mairéad Conneely ${ }^{1,2}$, Bláthín Casey ${ }^{3,4}$ and Susan Coote $1,4,5$

\begin{abstract}
Background: The implementation of condition-specific falls prevention interventions is proving challenging due to lack of critical mass and resources. Given the similarities in falls risk factors across stroke, Parkinson's Disease (PD) and Multiple Sclerosis (MS), the development of an intervention designed for groups comprising of people with these three neurological conditions may provide a pragmatic solution to these challenges. The aims of this umbrella review were to investigate the effectiveness of falls prevention interventions in MS, PD and stroke, and to identify the commonalities and differences between effective interventions for each condition to inform the development of an intervention for mixed neurological groups.
\end{abstract}

Methods: A systematic literature search was conducted using 15 electronic databases, grey literature searches and hand-screening of reference lists. Systematic reviews of studies investigating the effects of falls prevention interventions in MS, PD and stroke were included. Methodological quality of reviews was assessed using the A MeaSurement Tool to Assess Systematic Reviews 2. A matrix of evidence table was used to assess the degree of overlap. The Grading of Recommendations Assessments, Development and Evaluation framework was used to rate the quality of evidence. Findings were presented through narrative synthesis and a summary of evidence table.

Results: Eighteen reviews were included; three investigating effectiveness of falls prevention interventions in MS, 11 in PD, three in stroke, and one in both PD and stroke. Exercise-based interventions were the most commonly investigated for all three conditions, but differences were identified in the content and delivery of these interventions. Low to moderate quality evidence was found for the effectiveness of exercise-based interventions at reducing falls in PD. Best available evidence suggests that exercise is effective at reducing falls in stroke but no evidence of effect was identified in MS.

\footnotetext{
* Correspondence: Nicola.OMalley@ul.ie

'School of Allied Health, Faculty of Education and Health Sciences, University of Limerick, Limerick, Ireland

${ }^{2}$ Ageing Research Centre, Health Research Institute, University of Limerick, Limerick, Ireland

Full list of author information is available at the end of the article
}

(c) The Author(s). 2021 Open Access This article is licensed under a Creative Commons Attribution 4.0 International License, which permits use, sharing, adaptation, distribution and reproduction in any medium or format, as long as you give appropriate credit to the original author(s) and the source, provide a link to the Creative Commons licence, and indicate if changes were made. The images or other third party material in this article are included in the article's Creative Commons licence, unless indicated otherwise in a credit line to the material. If material is not included in the article's Creative Commons licence and your intended use is not permitted by statutory regulation or exceeds the permitted use, you will need to obtain permission directly from the copyright holder. To view a copy of this licence, visit http://creativecommons.org/licenses/by/4.0/ The Creative Commons Public Domain Dedication waiver (http://creativecommons.org/publicdomain/zero/1.0/) applies to the data made available in this article, unless otherwise stated in a credit line to the data. 
Conclusions: The findings suggest that exercise-based interventions are effective at reducing falls in PD, however, the evidence for MS and stroke is less conclusive. A strong theoretical rationale remains for the use of exercisebased interventions to address modifiable physiological falls risk factors for people with MS, PD and stroke, supporting the feasibility of a mixed-diagnosis intervention. Given the high overlap and low methodological quality of primary studies, the focus should be on the development of high-quality trials investigating the effectiveness of falls prevention interventions, rather than the publication of further systematic reviews.

Keywords: Parkinson's disease, Stroke, Multiple sclerosis, Falls, Umbrella review

\section{Background}

Up to $73 \%$ of stroke survivors fall in their first year poststroke and over half of people with Multiple Sclerosis (MS) (56\%) and Parkinson's Disease (PD) (59\%) experience a fall over a three- or six-month period, respectively [1-3]. Falls can result in physical injury for people with these neurological conditions, with research suggesting that between 11 and $17 \%$ of falls result in injury [4-6]. Notably, this figure was reported to be as high as $72 \%$ among stroke survivors [7]. Falls can have a number of psychosocial consequences including an increase in fear of falling and reduced self-efficacy [8], resulting in decreased independence and health-related quality of life $[9,10]$. Falls also lead to an increase in acute healthcare utilisation, higher home-care needs and/or greater institutional care needs $[5-7,11]$. Consequently, the development of effective evidence-based falls prevention interventions for people with MS, PD and stroke is a priority for research and service provision.

There are currently substantial shortcomings in the provision of services for people with neurological conditions in Ireland [12]. Despite the recent proliferation in the number of condition-specific falls prevention interventions being designed and evaluated, the implementation of these interventions in clinical practice is proving challenging for clinicians due to insufficient numbers of participants and resources to run group-based programmes [13]. Additionally, the stringent inclusion and exclusion criteria that are regularly associated with explanatory intervention trials reduce their transferability into clinical settings [14]. One potential solution to address these challenges is the development of an evidence-based pragmatic falls prevention programme that can be adapted for use among groups of individuals with different neurological conditions.

While there are differences in the pathophysiology and clinical presentation of stroke, PD and MS [15-18], people with these neurological conditions often present with a number of similar impairments and modifiable falls risk factors. Falls risk factors such as mobility impairments, decreased balance, strength deficits, cognitive dysfunction, depression and fear of falling, in addition to environmental and behavioural falls risk factors, are common across these conditions [19-28]. The mutual modifiable falls risk factors support a mixed-diagnosis intervention approach, as it is likely that the subsequent treatment approaches across the three conditions are similar. Moreover, differences in falls risk factors can exist in individuals with the same diagnosis, for example, not all people with PD present with freezing of gait [29]. Therefore, tailoring of a programme to a person's unique presentation is necessary for all interventions, independent of diagnosis, and is recommended in international guidelines for falls prevention [30,31]. Thus, it is anticipated that this model could also be used to develop a programme for people with MS, PD and stroke, and importantly be implemented in primary care. A mixeddiagnosis approach to the design and implementation of interventions could increase the number of eligible participants and services available. Consequently, the development of an intervention for groups with these mixed neurological conditions is timely to address implementation challenges in the community.

To date only one randomised controlled trial (RCT) has investigated the effect of a falls prevention intervention for people with MS, PD and stroke, reporting that an education programme did not reduce falls [32]. An umbrella review investigated the efficacy of conditionspecific, exercise-based falls prevention interventions for people with neurological conditions [33]. The review found exercise was effective at reducing falls among people with $\mathrm{PD}$, but insufficient evidence existed to determine their efficacy for people with stroke or MS [33]. The review focused on exercise only, however, a multimodal approach to falls prevention has been recommended to reduce falls risk [19]. Therefore, this umbrella review used a robust methodology to determine the effectiveness of all non-pharmacological and non-surgical falls prevention interventions for people with MS, PD and stroke, and compared and contrasted the effectiveness of interventions across these neurological conditions.

The objectives of this umbrella review were:

1. To summarise the totality of evidence regarding the effectiveness of non-pharmacological and nonsurgical falls prevention interventions for people with MS, PD and stroke. 
2. To identify commonalities and differences between interventions that are effective at reducing falls for people with MS, PD and stroke to inform the development of an evidence-based intervention that can be tailored for groups with mixed neurological conditions.

\section{Methods}

An umbrella review was conducted to identify and synthesise the results of systematic reviews (with or without meta-analysis) of studies investigating the effectiveness of falls prevention interventions at improving falls outcomes among people with MS, PD and stroke. In lieu of specific guidance for umbrella reviews, this umbrella review was conducted with reference to the Joanna Briggs Institute (JBI) Reviewer's Manual [34], the relevant sections of the Preferred Reporting Items for Systematic Reviews and Meta-Analysis (PRISMA) guidelines [35, 36], and the key aspects of methods and results of umbrella reviews outlined in the protocol for the Preferred Reporting Items for Overviews of Reviews (PRIOR) guidelines [37].

\section{Protocol and registration}

In compliance with best-practice recommendations to increase transparency and minimise bias, an a priori protocol for this umbrella review was developed [38]. This protocol was registered with the International Prospective Register of Systematic Reviews, PROSPERO, on the 28th April 2020 (CRD42020175409) and was published in an open access repository [39].

\section{Search strategy}

The authors developed a comprehensive search strategy to identify all pertinent research syntheses, both published and unpublished [40]. One reviewer (NO'M) completed searches of the following electronic databases: The Cochrane Database of Systematic Reviews, Joanna Briggs Institute Database of Systematic Reviews and Implementation Reports, Database of Abstracts of Reviews of Effects, PubMed, Embase, EBSCO (Academic Search Complete, AMED, Biomedical Reference Collection, CINAHL, Medline, PsycInfo, SPORTDiscus), Epistemonikos, PEDro and the PROSPERO register. All electronic databases were searched from date of inception to April 2020 (sample search string for the CINAHL is detailed in Appendix 1). In addition, the grey literature searches for relevant unpublished systematic reviews encompassed a search of OpenGrey and MedNar. Finally, the reference lists of all included systematic reviews were hand-searched to identify other potentially relevant reviews.

\section{Inclusion and exclusion criteria}

Quantitative systematic reviews (with or without metaanalysis), mixed-methods systematic reviews (quantitative elements only), or pooled analyses and research syntheses investigating the effectiveness of nonpharmacological falls prevention interventions for people with MS, PD and stroke were considered for inclusion in this umbrella review. Reviews published in the English language were included and authors of potentially relevant reviews published in a different language were contacted to ascertain if a copy of the review was available in English. No restriction was placed on year of publication of the review. In instances where a systematic review was an update of a previous review, the most recent version was included and the older version excluded. For the purposes of this umbrella review, a review was classified as an update of a previous version if there were changes pertaining to new data, new methods, or new analyses, but the research question, objectives and inclusion criteria remained similar [41]. In the case of new authors or a different research team updating an existing review, they had to clearly state that their review was an update and acknowledge the work of the authors on the previous edition [41].

The eligibility criteria based on population, intervention, comparator, outcome and study design are outlined below.

\section{Population}

We included reviews with adult participants ( $\geq 18$ years) with PD, MS or stroke according to a confirmed diagnostic criterion and reviews with a combination of these conditions. There were no exclusion criteria based on gender, disease duration, disease subtype or functional ability. For the purposes of this umbrella review, there was no exclusions based on the presence of comorbidities, however, when restrictions based on the presence of co-morbidities were a feature of included reviews, we extracted and recorded this.

\section{Intervention}

All non-pharmacological and non-surgical falls prevention interventions were included. Any intervention in which a primary or secondary aim was to reduce falls was considered a falls prevention intervention. Given the multifactorial nature of falls, and for inclusivity, there was no exclusion based on intervention content, intervention duration, intervention setting or mode of delivery of intervention.

\section{Comparator}

In instances where controlled trials were included in the systematic reviews all comparators were considered, 
including, but not limited to, usual care, enhanced care or waitlist control.

\section{Outcomes}

The primary outcomes of interest were any falls outcomes, measured as a primary or secondary outcome in the systematic reviews. For this umbrella review, the occurrence of a fall event had to be recorded in order to be classified as a falls outcome. This included, but was not limited to, total number of falls, falls rate, number of fallers, number of recurrent fallers or injurious falls. Of note, reviews in which falls were only measured as adverse outcomes were not included as the aim of the intervention was not to reduce falls. Additionally, reviews in which only laboratory-induced falls were recorded were excluded. Given that there is currently no consensus regarding what constitutes a fall, in addition to the variation in fall definitions present in the literature [42], a pre-determined definition for a fall event was not used in this umbrella review. Instead, all systematic reviews were considered for inclusion regardless of their definition of a fall, but these definitions were extracted and presented to help readers contextualise the results.

Secondary outcomes of interest were those relating to the effectiveness and implementability of interventions. Secondary outcomes were only extracted in instances where falls were measured as a primary outcome and where it was possible to extract this data for the populations and interventions of interest.

\section{Study design}

Systematic reviews of all study designs investigating falls prevention interventions were considered for inclusion.

Potentially relevant papers were screened for inclusion as a systematic review by two independent reviewers (NO'M and BC) using the JBI Critical Appraisal Checklist for Systematic Reviews and Research Syntheses [34]. Any disagreements between reviewers were resolved through discussion or through consulting a third reviewer until consensus was achieved. Any review that received a 'No' response to Items $2,3,4,5$ or 8 were excluded [43, 44].

\section{Study selection}

The citations yielded from the searches were exported to the master reference management library Rayyan, where duplicate papers were then removed. The titles and abstracts were screened by two independent reviewers (NO'M and BC) against the eligibility criteria. The authors of potentially relevant protocols and conference abstracts were contacted to determine the full text publication status. Following this, the full texts of potentially relevant reviews were obtained and screened for eligibility by two independent reviewers (NO'M and BC). Any discrepancies between reviewers were resolved through discussion or through consultation with a third reviewer until consensus was achieved.

\section{Dealing with overlap of primary studies}

Overlap of primary studies within included systematic reviews is a challenge exclusive to umbrella reviews. Currently, there is a lack of guidance on how best to manage this occurrence [45]. To maximise comprehensiveness of this umbrella review, we employed a 'full inclusion scenario' where all Cochrane reviews and nonCochrane reviews were included [46]. A list of the primary studies included in each systematic review was assembled and a matrix of evidence table was created to determine the amount of overlap between systematic reviews. To avoid double-counting outcome data the following were decided:

- Any systematic review that contained a relevant primary study that was not in any other systematic review was included so that data were not lost.

- Any systematic review that did not contain any unique primary study (i.e. a primary study not already present in an included review) was excluded to minimise duplication of data.

- In the presence of complete overlap between reviews, the highest quality review, as determined by the A MeaSurement Tool to Assess Systematic Reviews 2 (AMSTAR 2) was included in data synthesis and analysis.

- In cases where there was a complete overlap between reviews and they received the same AMSTAR 2 rating, then the most recently published review was included.

- In the presence of partial overlap, all reviews were included but the degree of overlap was noted and its implications on the findings of this umbrella review were discussed.

\section{Methodological quality assessment}

Two independent reviewers (NO'M and BC) assessed the methodological quality of included reviews using the AMSTAR 2 [47]. In line with recommendations, the critical domains for the AMSTAR 2 were classified as Items 2,4,7,9,11,13 and 15 [47]. The overall score of the AMSTAR 2 was used to rate the quality of each included review as high, moderate, low or critically low [47] [47]..

It has been proposed that the use of the PRISMA reporting guidelines, in addition to a comprehensive, validated quality appraisal tool, facilitates the judgement of not only the methodological quality but also the general quality of reporting of included systematic reviews [48]. Therefore, the full texts of all systematic reviews included in this umbrella review were cross-checked 
against the PRISMA reporting guidelines checklist [35, 36].

\section{Data extraction}

Data were extracted by one reviewer (NO'M) using the JBI standardised data extraction form for umbrella reviews [34]. The following were also considered key data to answer our research question and were extracted where available: the definition of a fall, the faller classification and the person delivering the intervention. This form was then checked by a second reviewer (MC) to ensure that the extracted data were accurate. Disagreements regarding data extraction were resolved through discussion or by consulting a third reviewer until consensus was achieved.

\section{Quality of evidence}

The quality of evidence was assessed using the Grading of Recommendations Assessments, Development and Evaluation (GRADE) algorithm that has been developed for application to umbrella reviews [49]. The GRADE algorithm was applied to the included systematic reviews by two reviewers (NO'M and MC) to assess the quality of the evidence relating to the following outcomes:

1. Total number of falls - the number of falls recorded by participants throughout the study period

2. Falls rate - the number of falls per person per specific period of time, e.g. falls per person per year

3. Number of fallers - the proportion of participants classified as 'fallers' based on the criteria outlined by the researchers e.g. an individual who has one or more falls during the follow-up period

\section{Data synthesis and analysis}

Given the heterogeneity in populations, outcomes and analyses, the findings of included reviews were primarily summarised using a narrative synthesis with the quantitative tabulation of results as appropriate. The primary analyses for this umbrella review were centred on type of neurological condition and type of intervention. Following this, cross-comparison of similarities and differences in the effect of different interventions between the three conditions were reported and discussed. The outcomes of each included systematic review were considered and discussed in the context of their methodological quality, as determined by the AMSTAR 2 and the GRADE algorithm.

\section{Discordance between reviews}

Umbrella reviews provide an opportunity for researchers to address the issue of discordance between reviews and to identify its cause [48]. In instances of discordant reviews in our umbrella review, the algorithm developed by Jadad et al. (1997) was used to resolve and discuss issues of discordance [50].

\section{Deviations from protocol}

To facilitate comparison of intervention effectiveness, we had planned to have a standardised approach to our results by converting the different estimates of effect that we extracted to one common effect measure. However, these analyses were not possible due to the small number of meta-analyses and the heterogeneity between studies in terms of outcomes assessed. We had anticipated that many of our included systematic reviews would include non-randomised trials and as a result had planned to examine the effects of synthesising reviews of varying quality, however, this was not the case as only three systematic reviews included non-randomised trials and so this analysis was not completed.

\section{Results}

Figure 1 illustrates the study selection process for this umbrella review. A total of 51 reviews were deemed to have met our inclusion criteria. The reasons for exclusion of reviews following full-text screening are reported in Additional file 1. A citation table was produced to establish the degree of overlap between these reviews (see Additional file 2), thus excluding 33 systematic reviews using the predefined criteria for dealing with overlap. Eighteen systematic reviews were included in the final synthesis; three reviews including people with MS [5153], three reviews including people with stroke [54-56], 11 reviews including people with PD [57-67] and, finally, one review including primary studies with people with stroke and PD [68]. The matrix of evidence table outlining the final citation count and the degree of overlap for these 18 included reviews is presented in Table 1.

\section{Characteristics of included systematic reviews}

Specific details regarding the characteristics of the 18 included reviews are presented in Additional file 3. The included systematic reviews were published between 2010 and 2020. Of those systematic reviews, nine conducted meta-analyses [51, 53-56, 63, 64, 66, 68], however, only six of those reviews conducted a meta-analysis on a falls outcome $[51,54,56,63,64,68]$. The remaining 12 presented their findings on falls outcomes through a narrative synthesis. Falls data were extracted from RCTs only in 15 included reviews, a combination of RCTs and nonrandomised studies of intervention (NRSIs) in two reviews, and NRSIs only in one systematic review. The included systematic reviews comprised of primary studies conducted between 1990 and 2018. 17, 24 and 32 primary studies informed the falls outcomes of systematic reviews including people with MS, people with stroke 


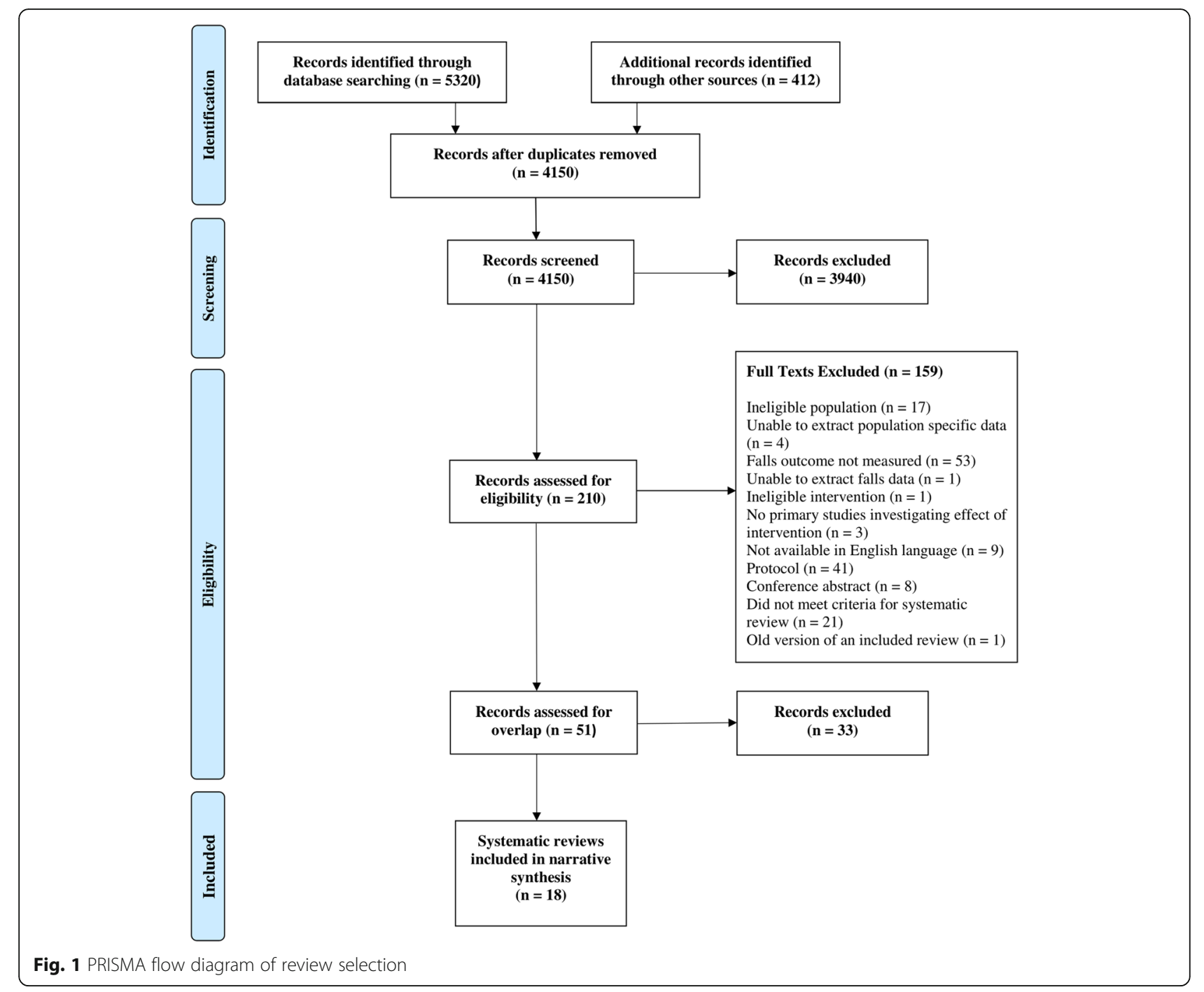

and people with PD, respectively. Despite the exclusion of 33 systematic reviews, some overlap of primary studies across included reviews remained, as demonstrated in Table 1.

\section{Methodological quality}

The methodological quality of included systematic reviews is presented in Fig. 2. The quality of included reviews ranged from critically low to moderate, with no included review rated as high quality. The majority of reviews $(n=12)$ were rated as critically low $[52,53,56-64$, 67], with three systematic reviews rated as low $[55,66$, $68]$ and three rated as moderate [51, 54, 65]. None of the included systematic reviews reported the sources of funding for included primary studies. The majority of those rated low or critically low were deemed to have critical flaws relating to a priori protocol development, a comprehensive search strategy, and the listing and justification for exclusions.

\section{Reporting quality}

In general, the reporting across included reviews was relatively complete, however, as shown in Table 2, there were some reporting flaws identified. Firstly, nine of the included reviews did not identify the paper as a systematic review and/or meta-analysis in the title and 11 of the reviews did not register an a priori protocol. This umbrella review also found inadequate reporting of the search strategy, data items, summary measures, results of individual studies and funding.

\section{Participant characteristics}

The number of participants in included reviews ranged from 21 to 1358. The mean age of participants was lower in systematic reviews of people with MS (range 36-63 years in included primary studies) compared to systematic reviews of people with PD and stroke, where the mean age of participants was greater than 60 years in the vast majority of included primary studies. Disease 


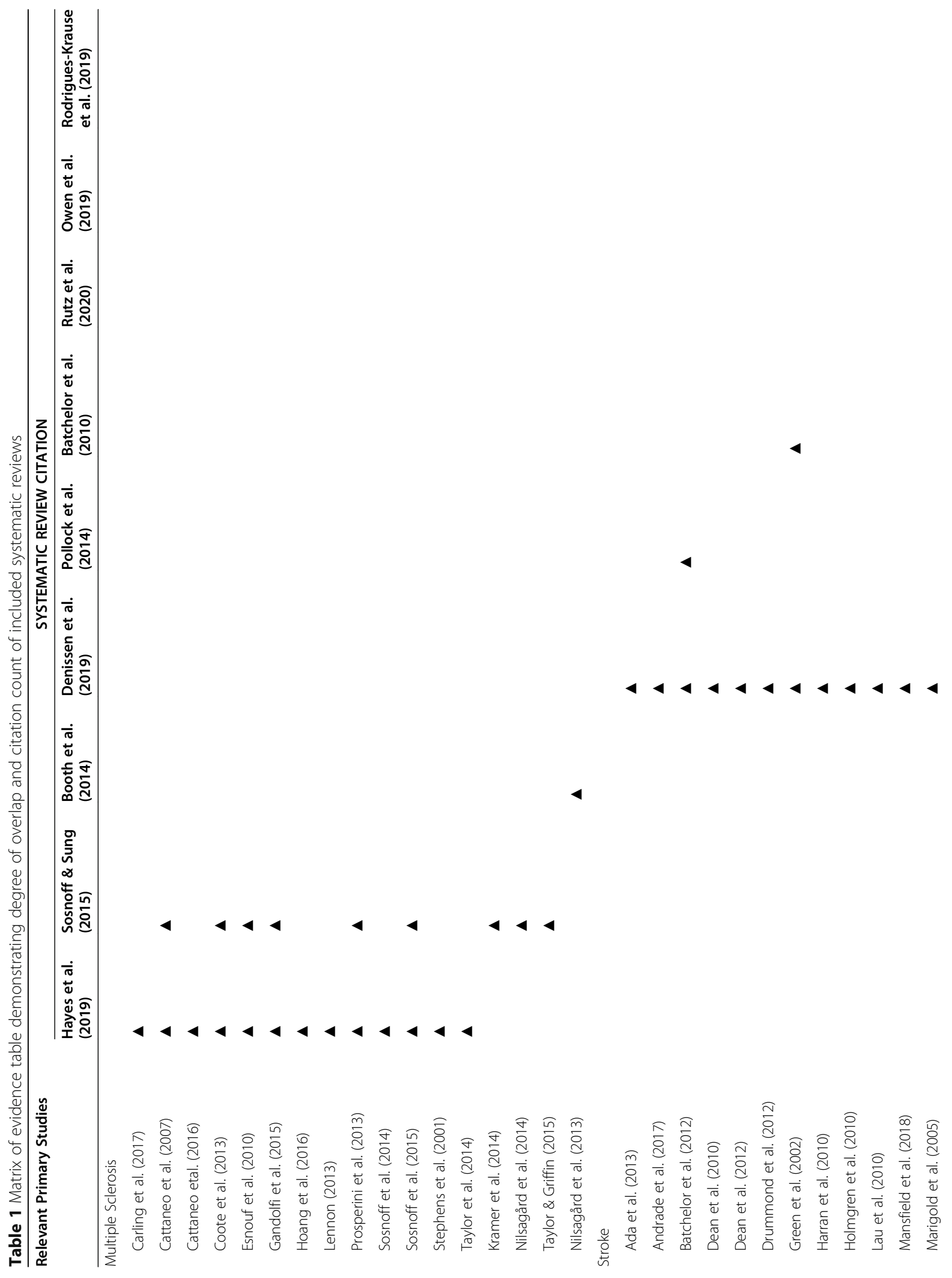




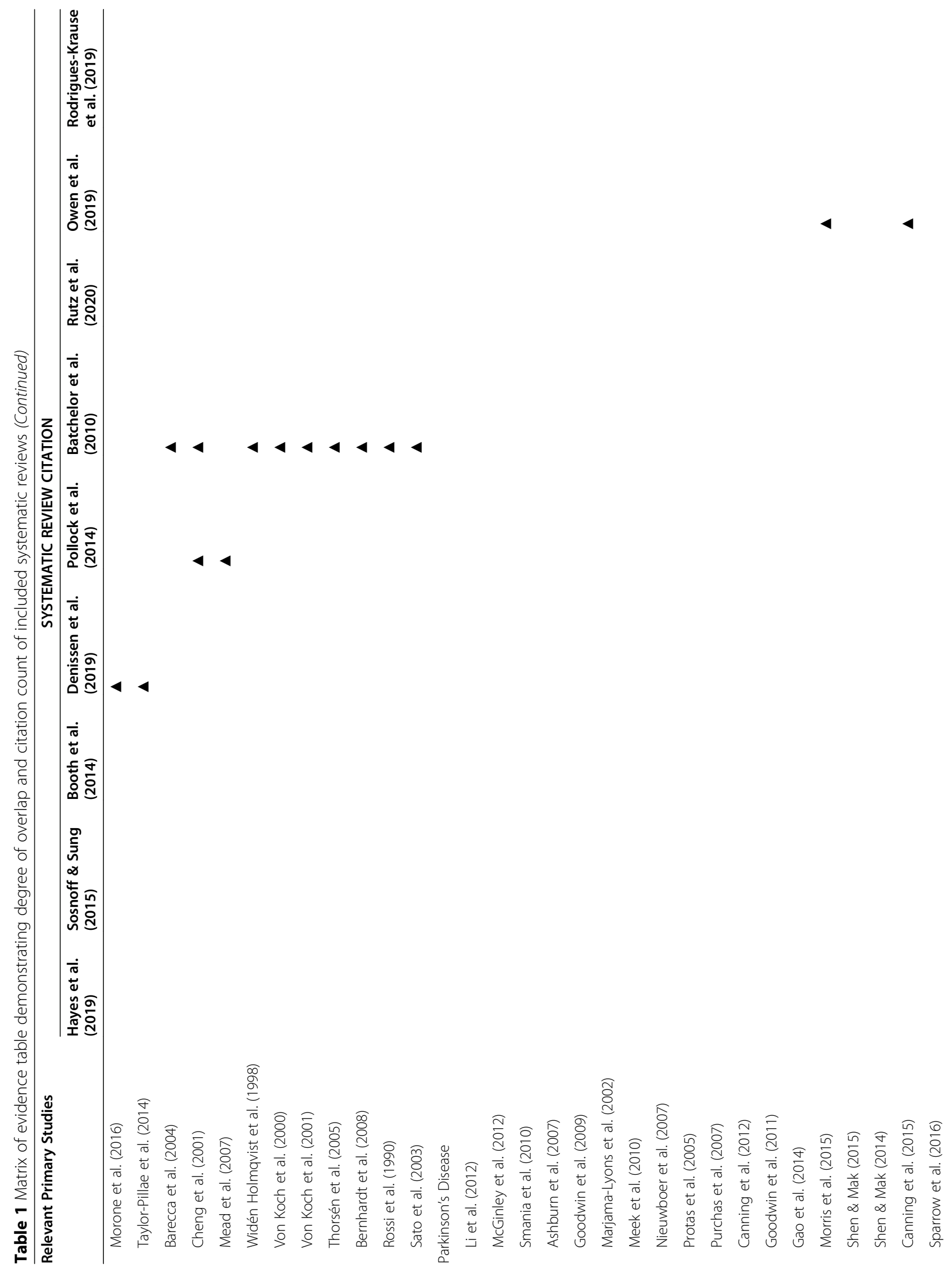




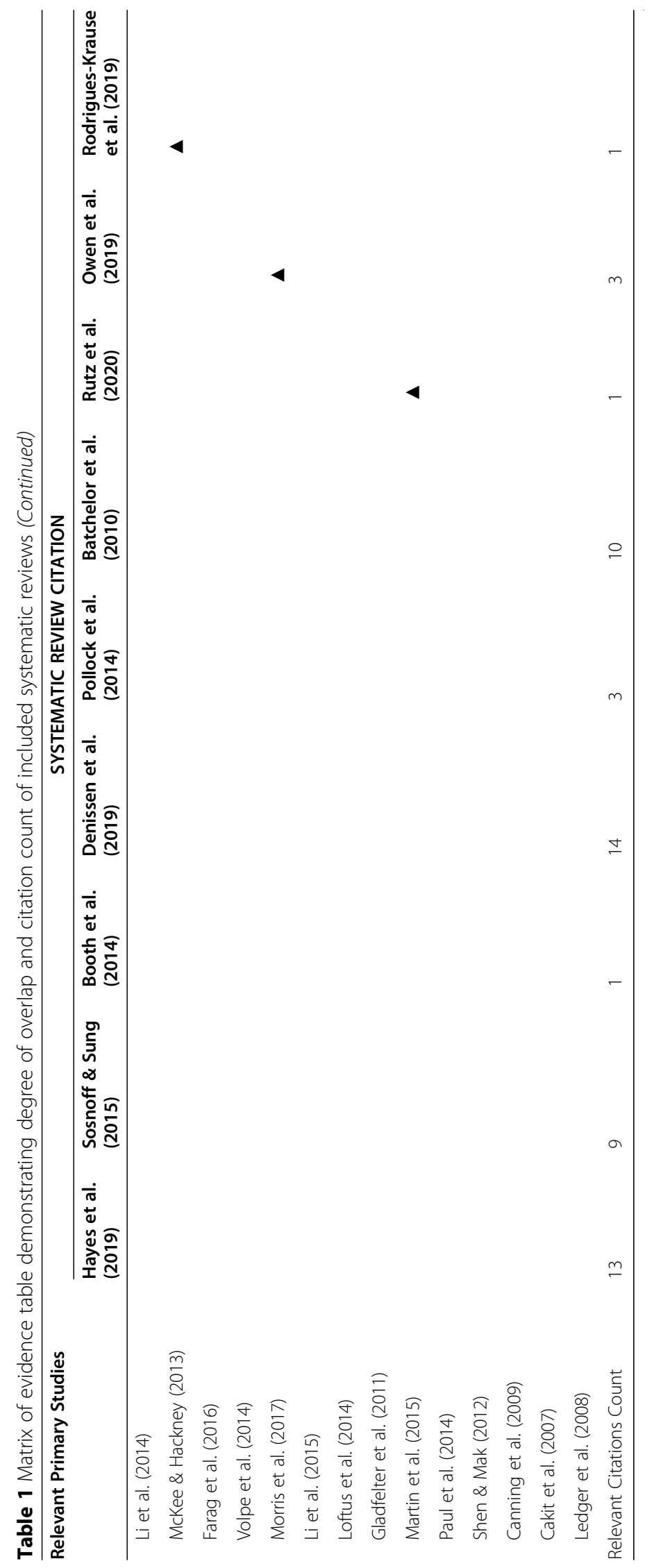




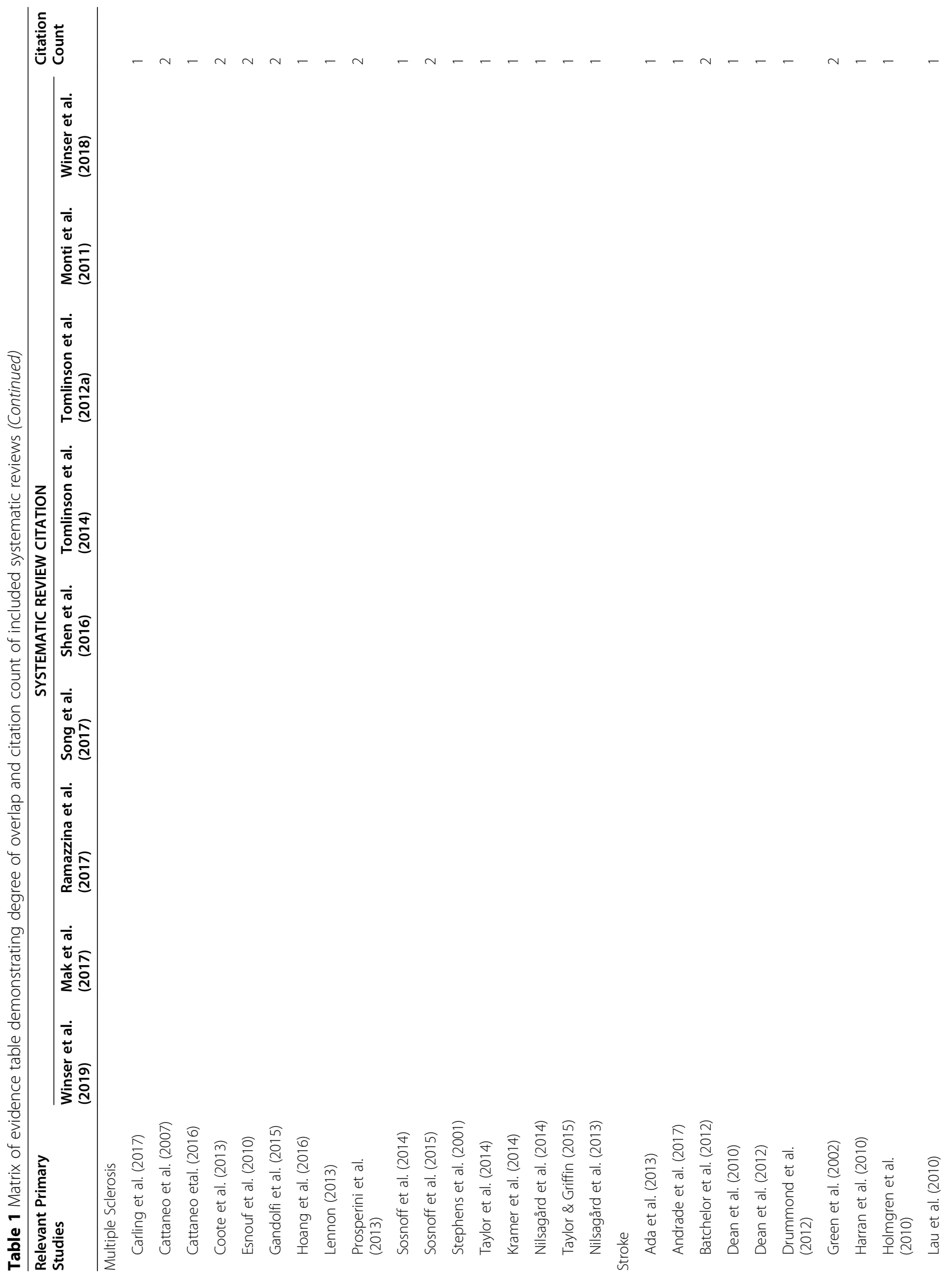




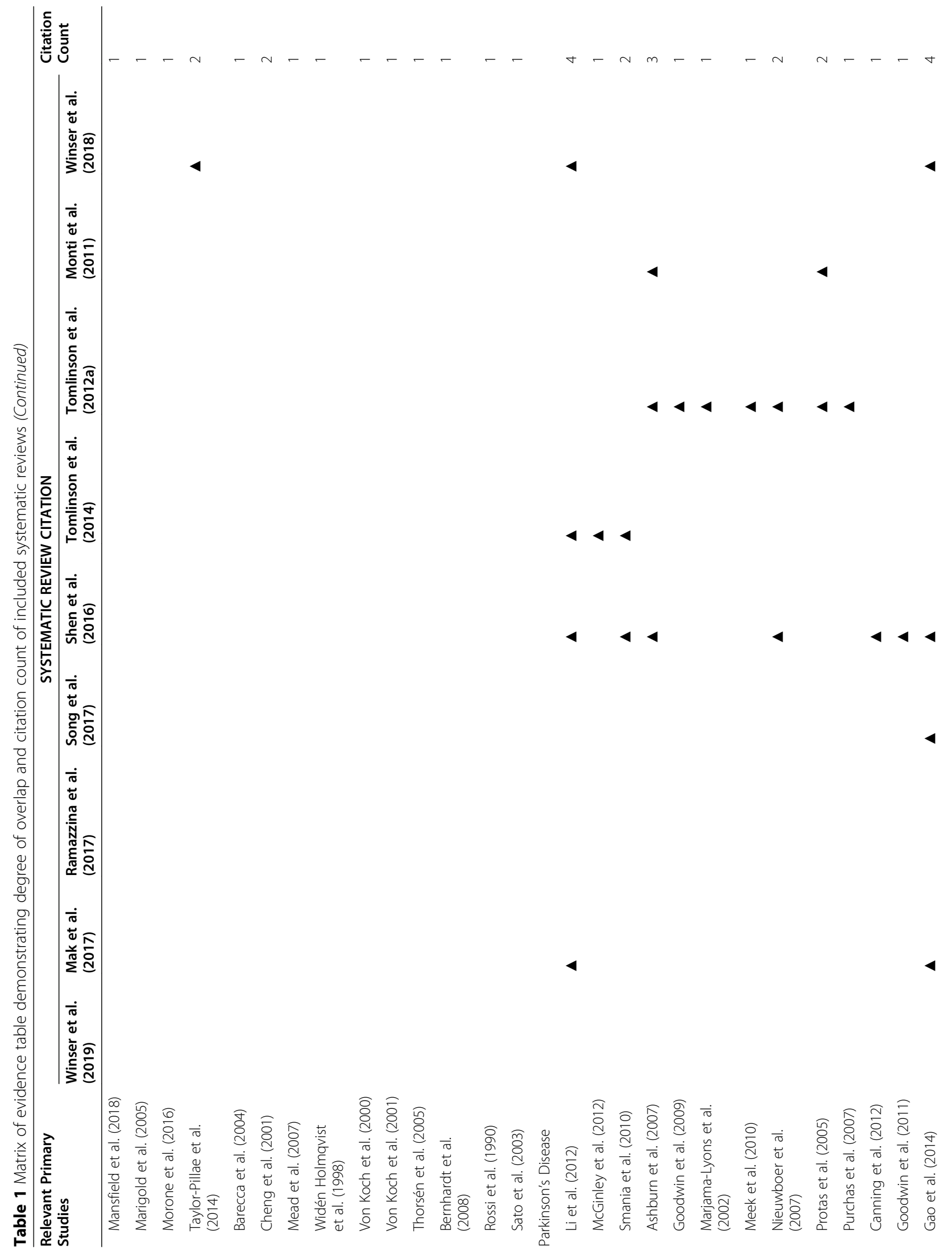




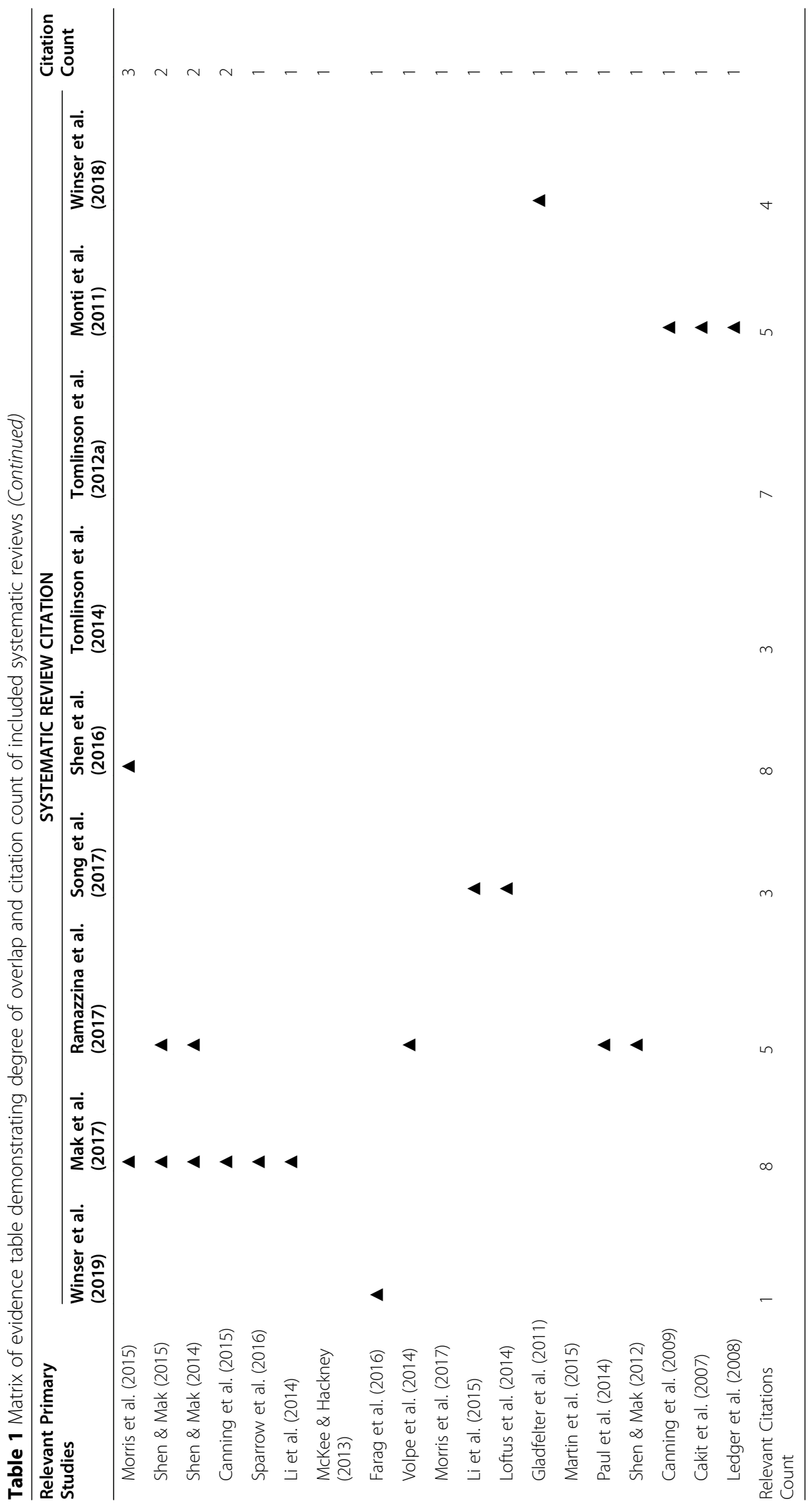




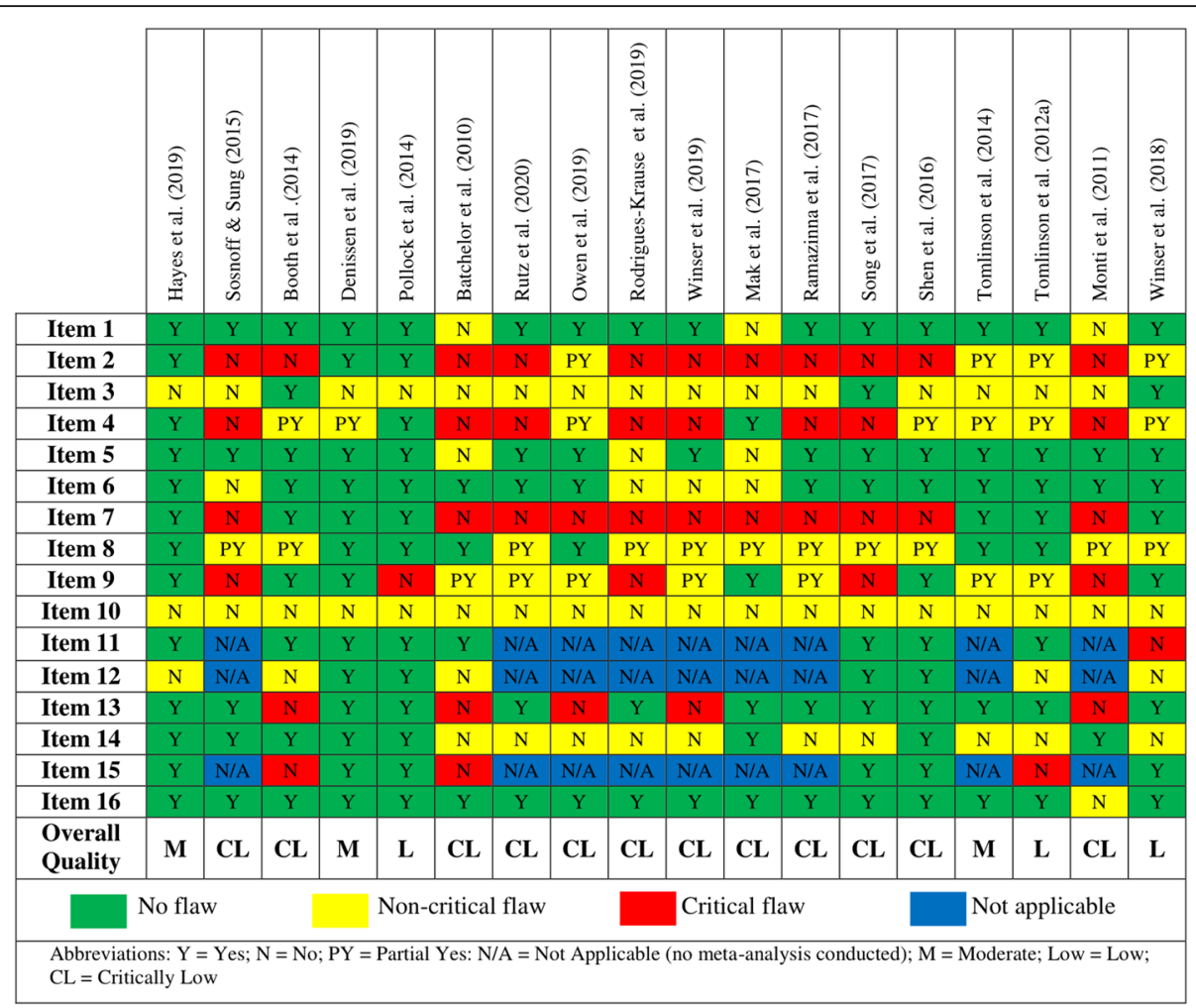

Fig. 2 AMSTAR 2 ratings of included systematic reviews

severity and functional ability were not regularly reported in included reviews but, of note, when it was participants generally had a relatively low disease severity and high functional level. Specific details regarding the participants in each included systematic review are presented in Table 3 and Additional file 3.

\section{Critical appraisal of primary studies}

As presented in Additional file 3, a variety of different critical appraisal instruments were used to assess the methodological quality of included primary studies. The methodological quality of primary studies was varied and was noted as a limitation in the majority of included systematic reviews. A detailed summary of the critical appraisal of the primary studies is outlined in Additional file 3.

\section{Person delivering the intervention and intervention setting}

The person delivering the intervention and intervention setting were not regularly reported in included systematic reviews. Of those presenting the person delivering the intervention, physiotherapists were most common, other disciplines included occupational therapists, yoga instructors, Tai Chi instructors, optometrists and multidisciplinary teams. Interventions were primarily delivered in the community or the participants' homes, but other settings including rehabilitation centres, hospitals and acute care were also reported.

\section{Intervention characteristics}

Exercise-based interventions were the most common across all three conditions, included in a total of 15 systematic reviews. Tai Chi and treadmill walking interventions were assessed among people with PD and stroke, but not MS. Dance-based exercise interventions were only investigated among people with PD. Technologybased interventions and multicomponent interventions were reported across more than condition, with all other reported interventions investigated amongst only one. There was substantial variation across reviews with respect to the reporting of intervention details. Specific intervention characteristics including, where reported, content, dose and duration are presented for included reviews in Table 3 and Additional file 3.

\section{Exercise-based interventions}

This umbrella review identified low to moderate quality evidence for exercise-based interventions among people with PD, with seven out of ten reviews reporting a significant effect of intervention on the recorded falls outcome [59-61, 63, 64, 67, 68]. The remaining three reviews for PD identified mixed results regarding the effectiveness of intervention across included primary 
O'Malley et al. BMC Neurology

(2021) 21:378

Page 14 of 31

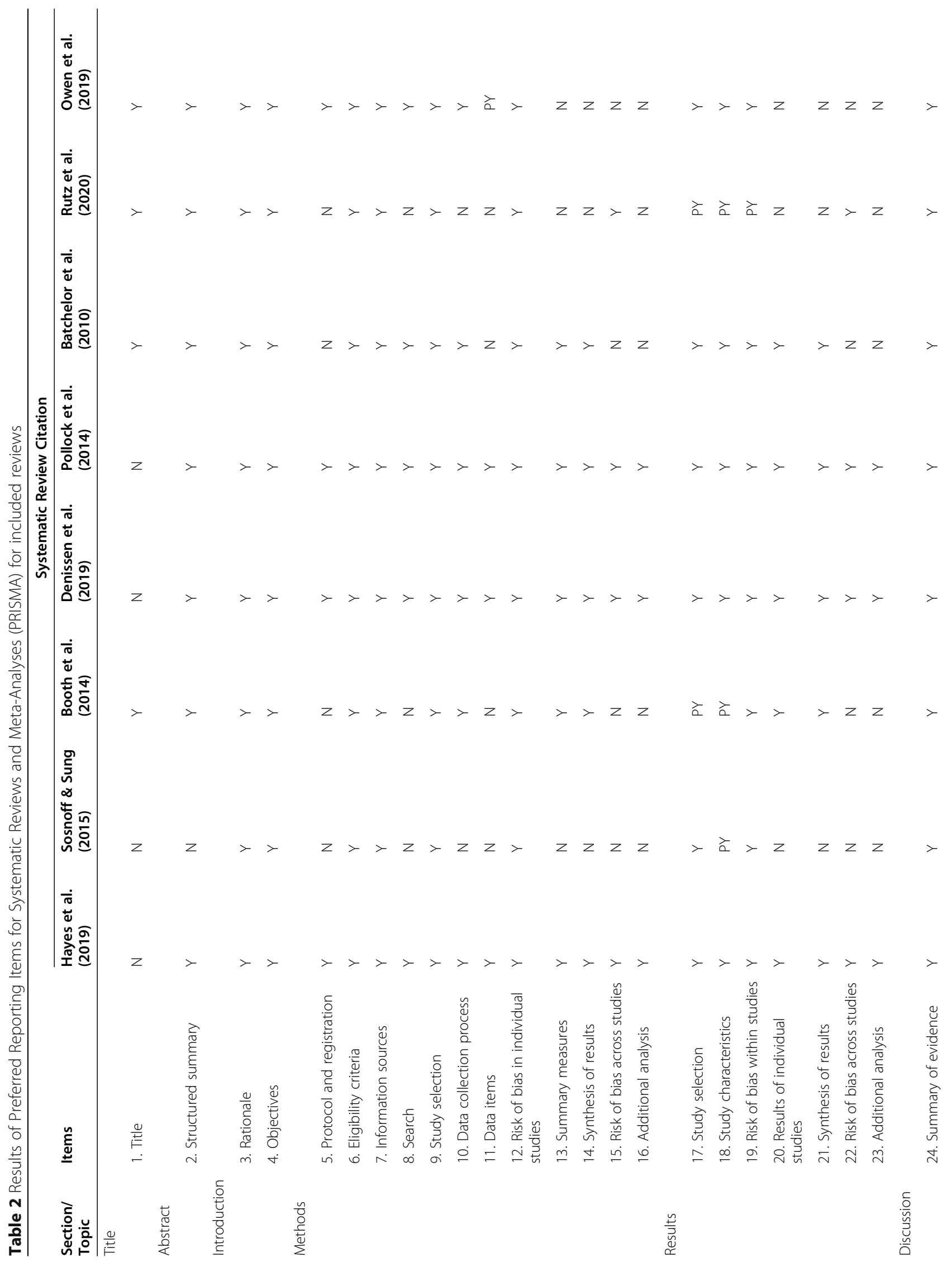




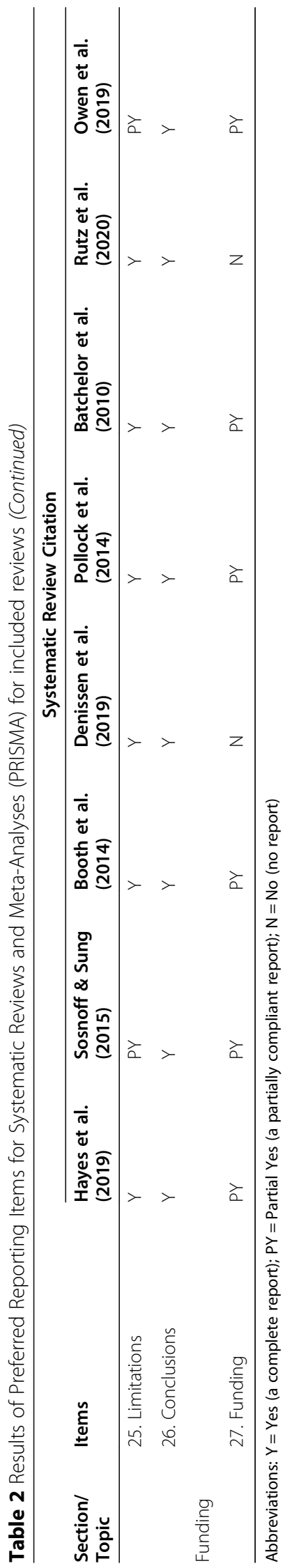


O'Malley et al. BMC Neurology

(2021) 21:378

Page 16 of 31

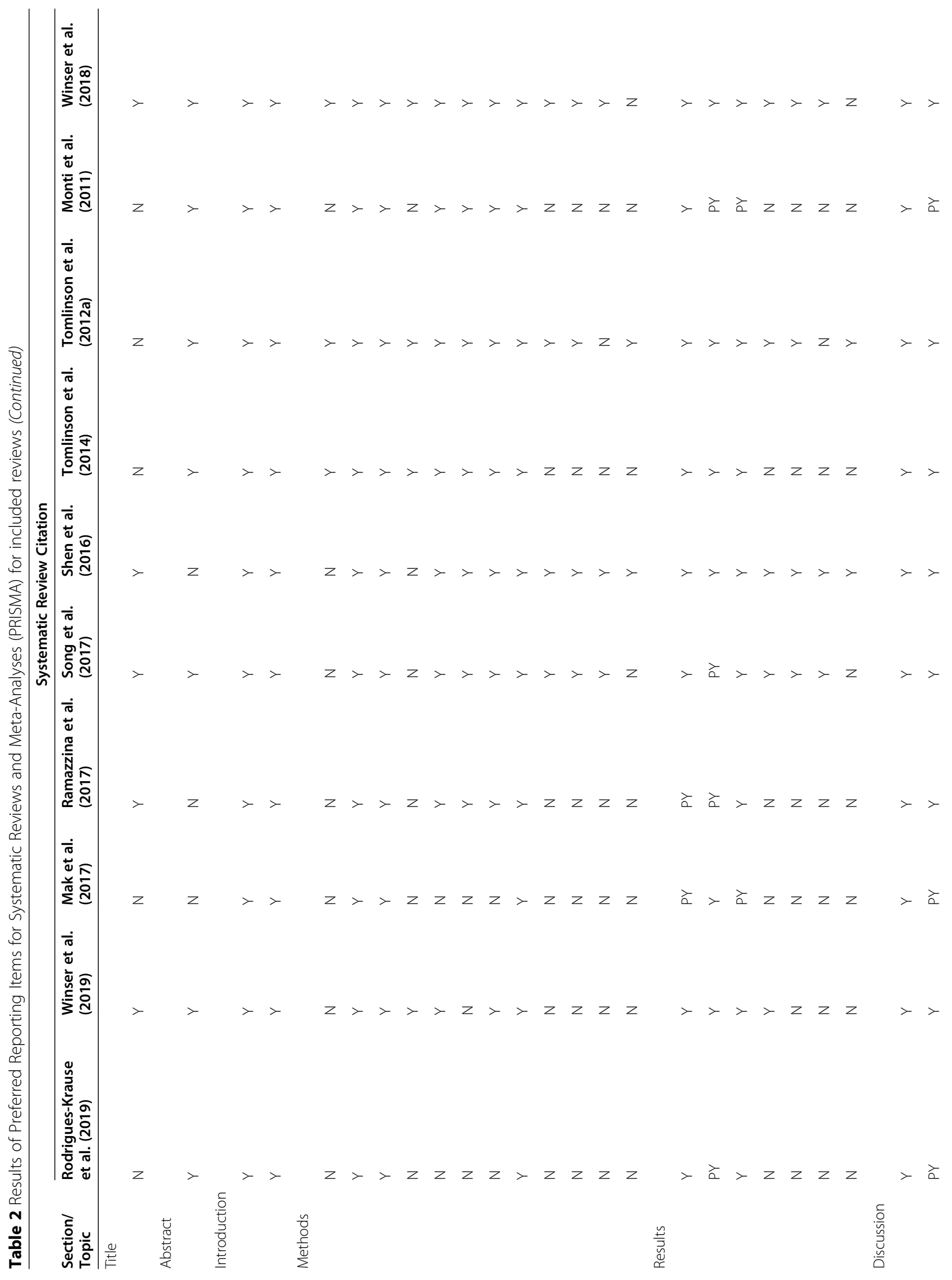


O'Malley et al. BMC Neurology

(2021) 21:378

Page 17 of 31

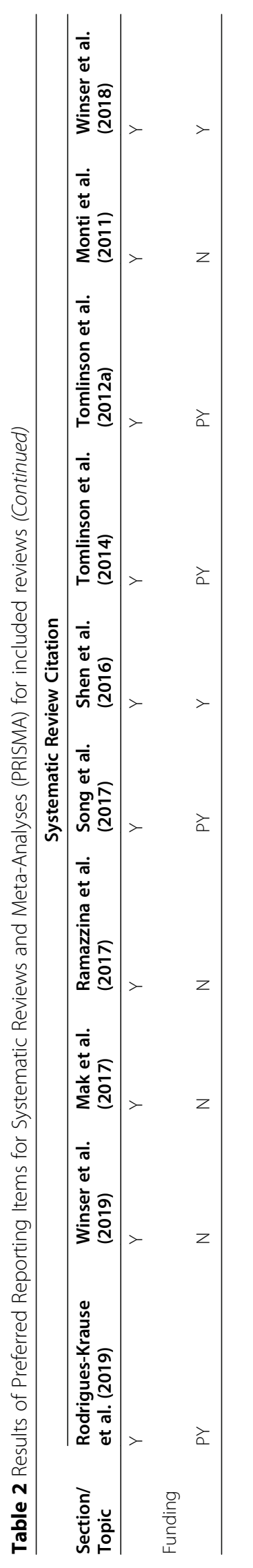




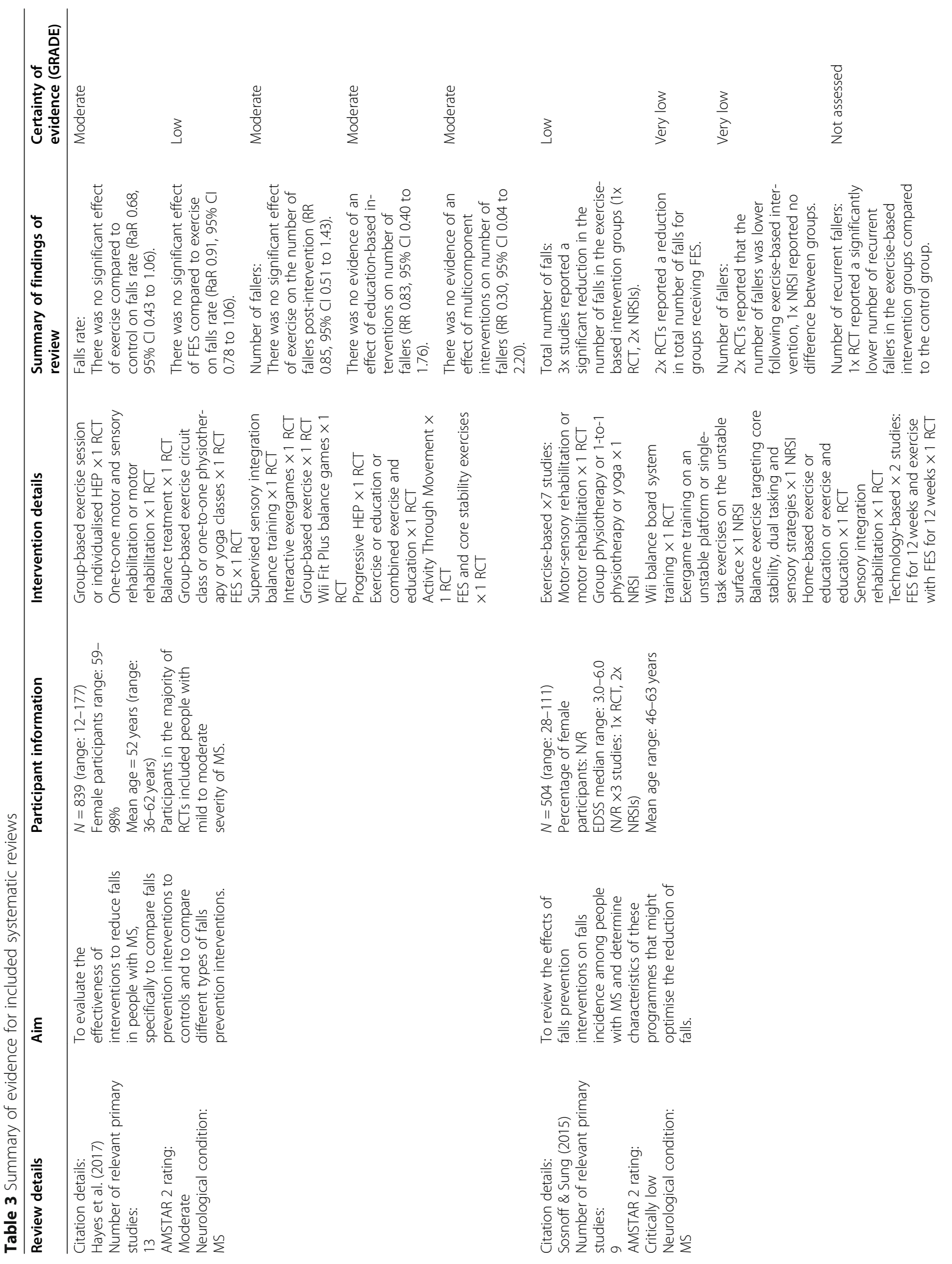




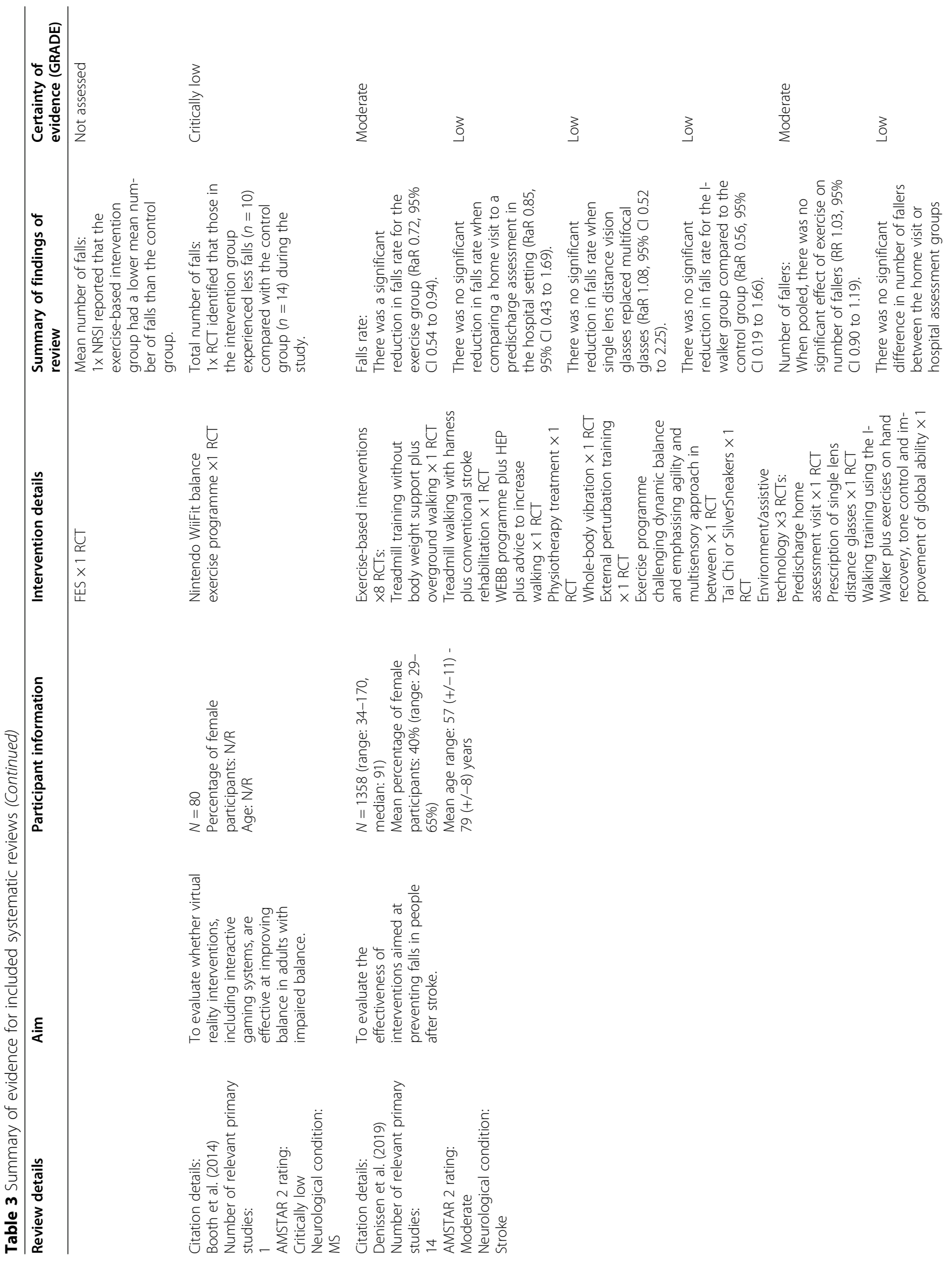




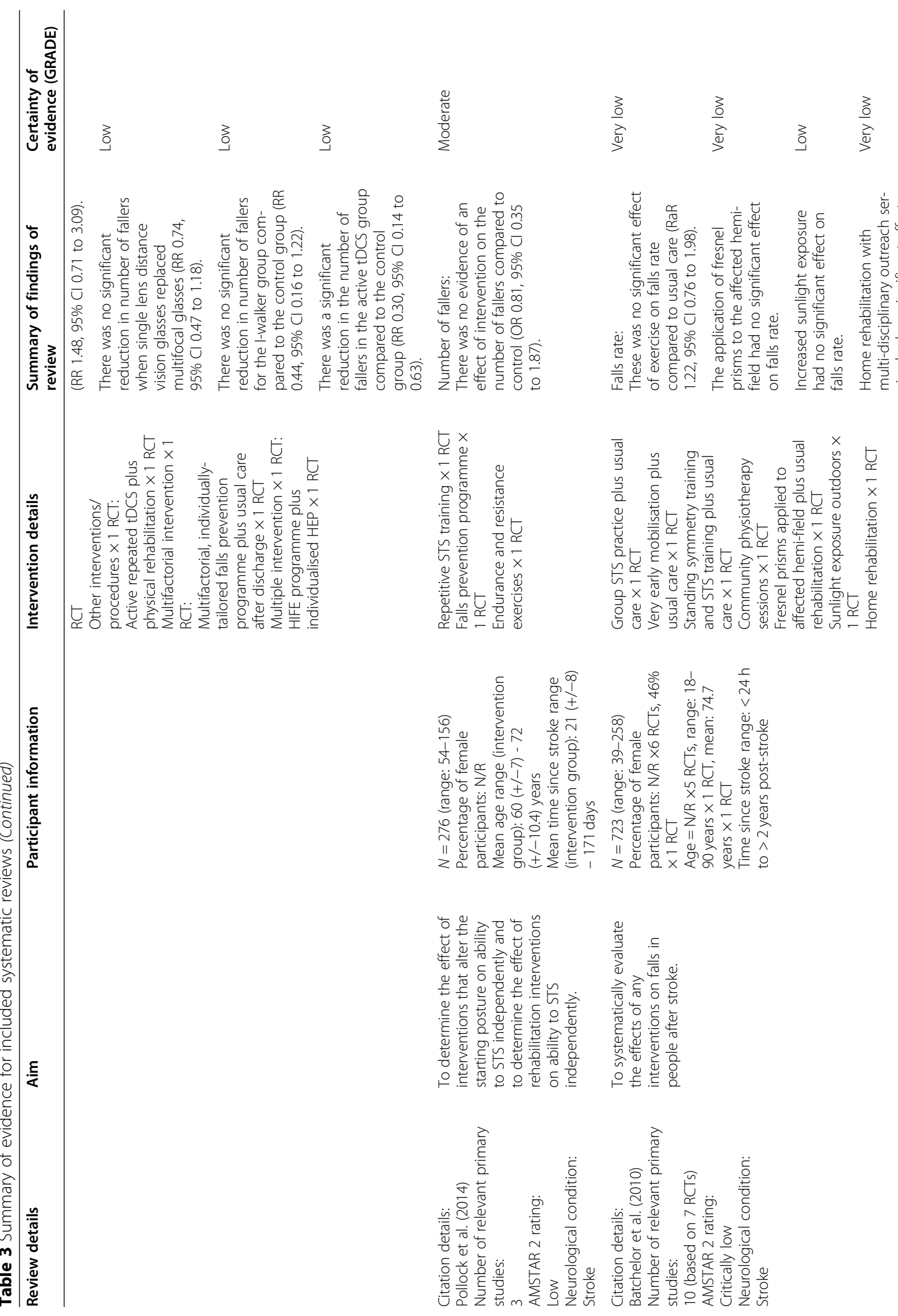




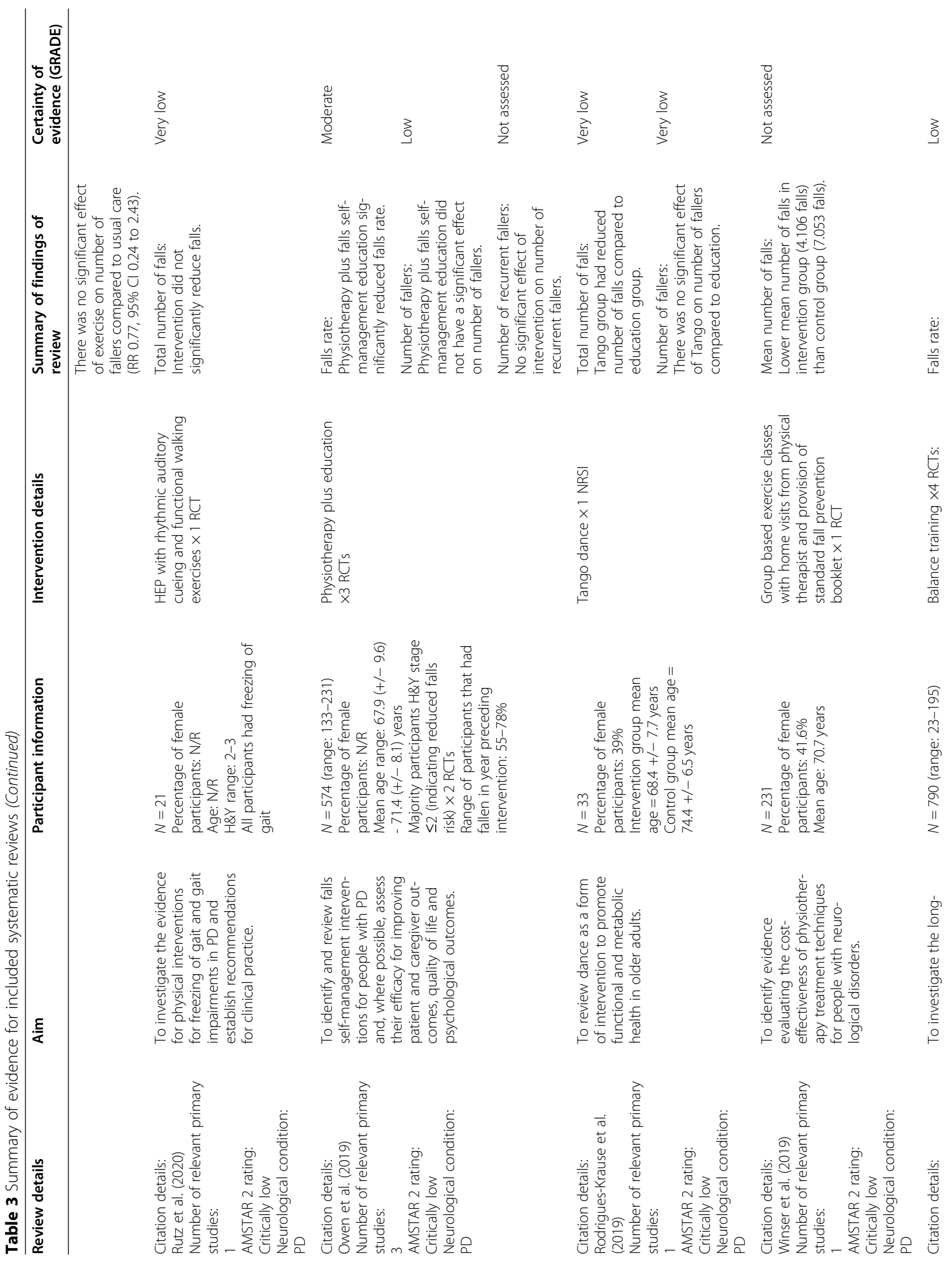




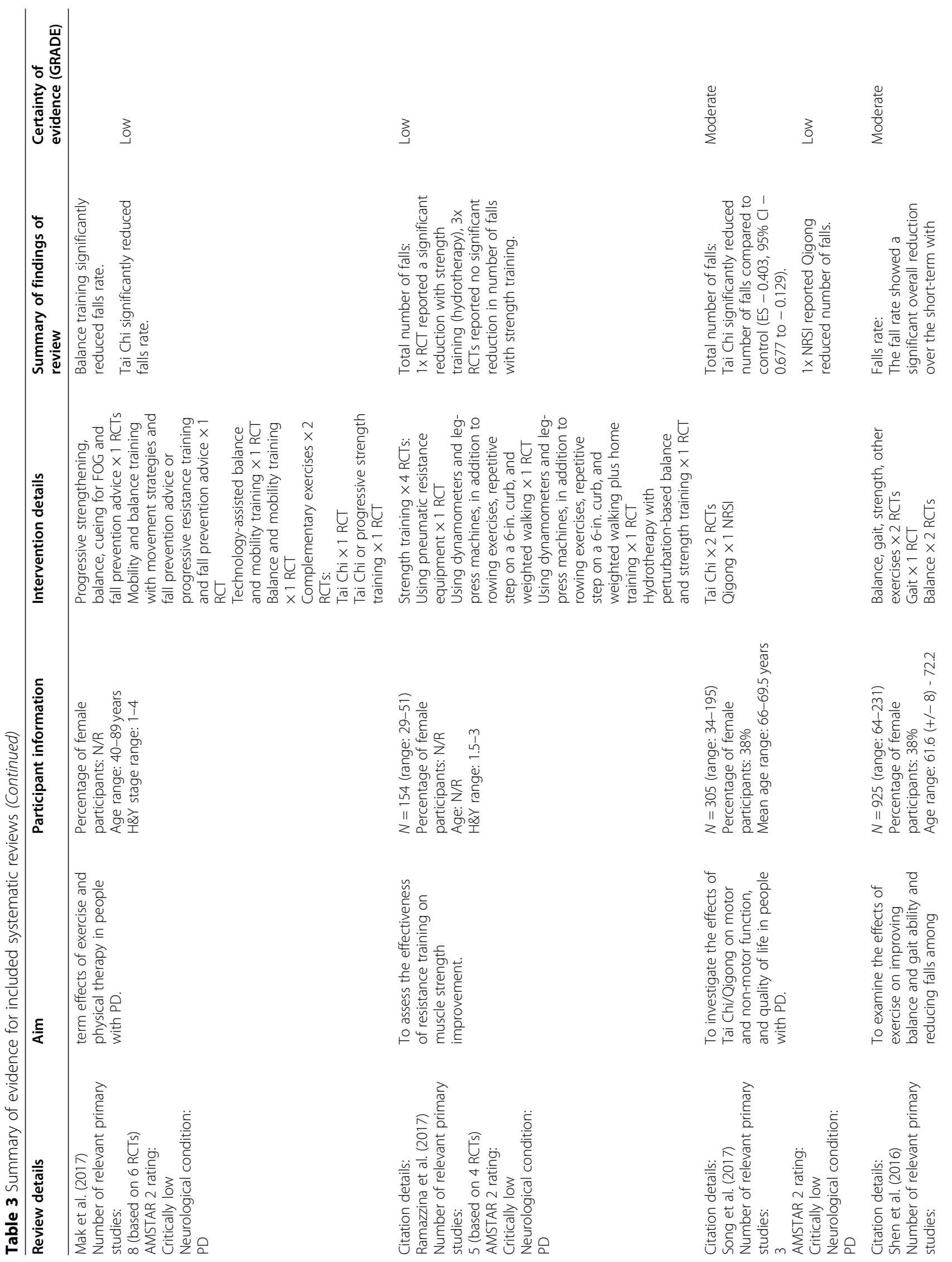




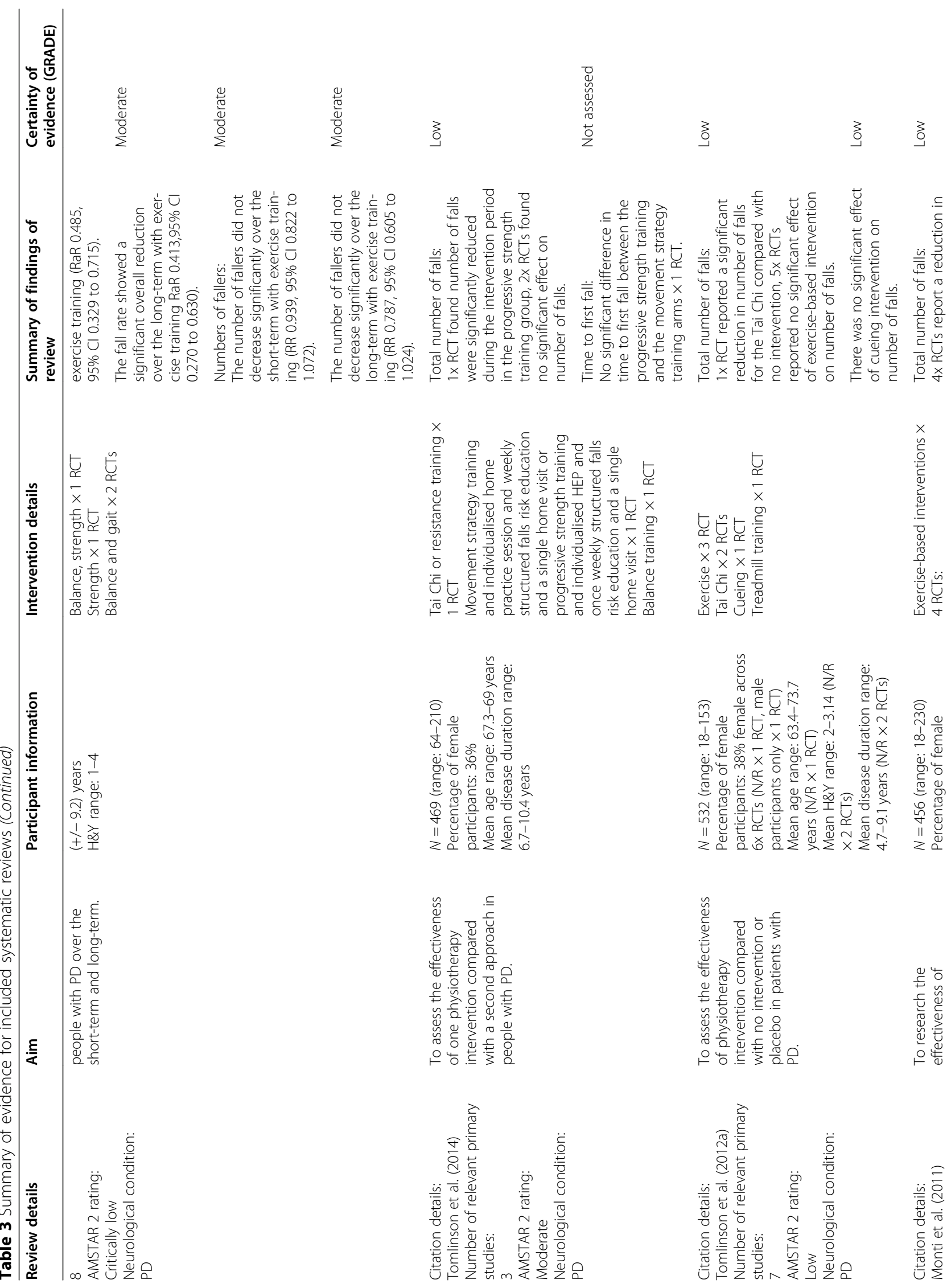




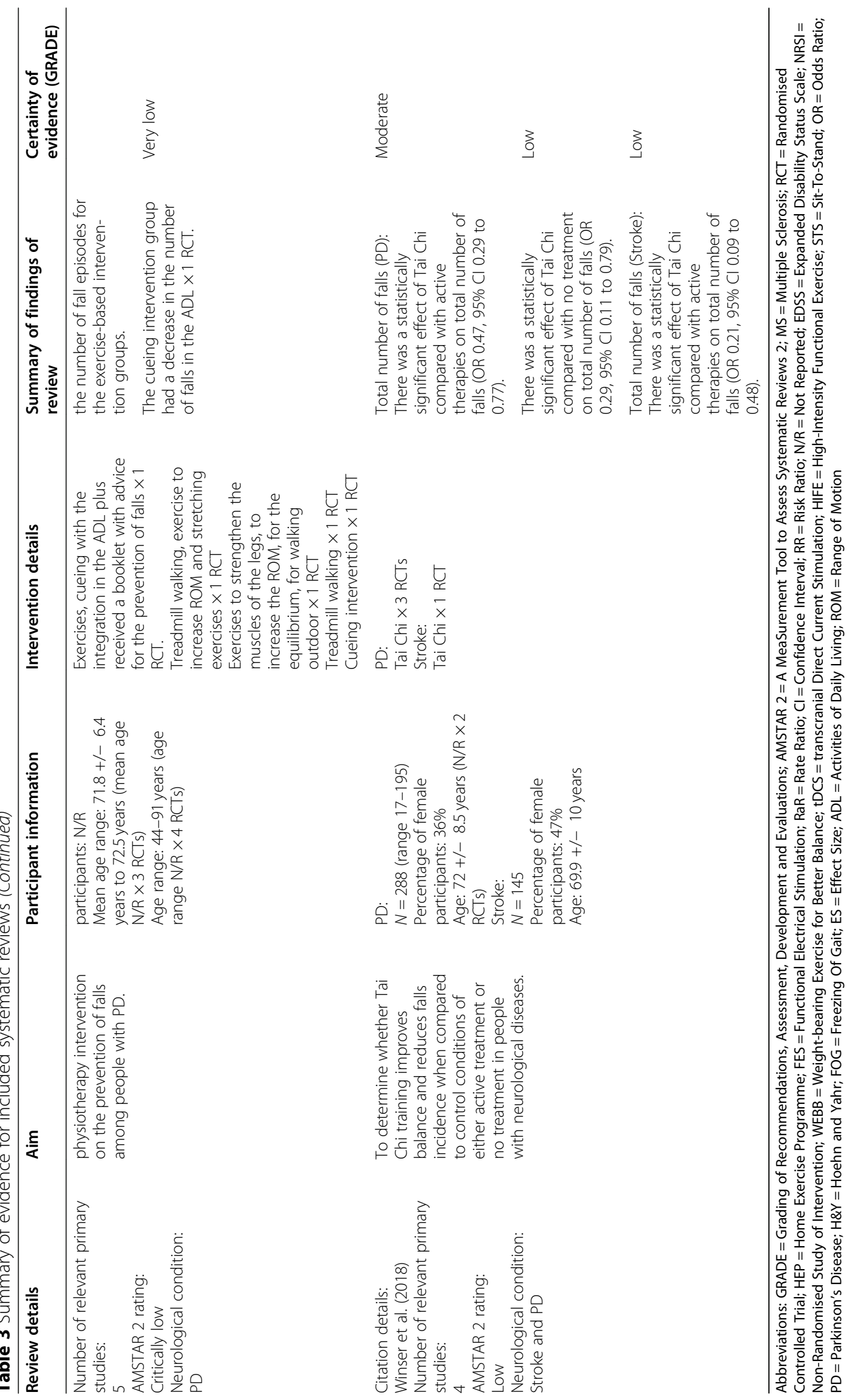


studies [62, 65, 66]. The effectiveness of exercise-based interventions varied across reviews for people after stroke, with two reviews reporting significant improvements in falls outcomes [54,68] and two reporting no evidence of effect [55, 56]. However, the two that showed effect were of higher methodological quality and provided low to moderate quality evidence for the effectiveness of exercise at improving falls outcomes for people after stroke. The evidence for exercise among people with MS also differed across reviews with one reporting no effect [51] and two reporting an improvement in falls outcomes $[52,53]$, but the most recently published review that had the highest methodological quality and the largest number of primary studies informing outcomes reported no evidence of effect of exercise-based interventions on falls rate or number of fallers.

\section{Technology-based interventions}

Technology-based interventions were investigated among people with MS and stroke. The evidence regarding the effectiveness of these interventions varied across reviews for people with MS, with one review of moderate methodological quality reporting no significant effect of functional electrical stimulation (FES) [51] compared to another of critically low methodological quality that reported FES led to a reduction in total number of falls and number of fallers [52]. Technology-based interventions were investigated in one systematic review for people with stroke, providing low quality evidence for transcranial direct stimulation to reduce number of fallers [54].

\section{Multicomponent and multifactorial interventions}

Multicomponent interventions were investigated for people with MS and PD in one systematic review each $[51,58]$. No significant effect of multicomponent interventions was identified for people with MS or PD on number of fallers. However, there was moderate evidence identified for the effectiveness of a multicomponent intervention comprising of physiotherapy and fallsself management education at reducing falls rate among people with PD [58].

Two systematic reviews for people with stroke included a multifactorial intervention [54, 55], however, this intervention was primarily comprised of exercises and so was included in the analyses for exercise-based interventions in these reviews.

\section{Education-based interventions}

Education interventions were only reported in one systematic review of moderate methodological quality among people with MS [51]. This review concluded that there was no significant effect of education-only interventions on number of fallers but this outcome was only informed by one primary study.

\section{Environment/assistive technologies}

Environment/assistive technologies were assessed among people with stroke in two reviews ranging from critically low to moderate methodological quality $[54,56]$. These reviews identified no significant effect of intervention on number of fallers or falls rate.

\section{Cueing interventions}

Cueing interventions for people with PD were assessed in three systematic reviews of low to critically low methodological quality $[57,66,67]$. One of these reviews provided very low quality evidence for the effectiveness of cueing at reducing falls [67]. The two remaining systematic reviews reported that there was no significant effect of cueing interventions on total number of falls $[57,66]$.

\section{Interventions to improve bone mineral density}

One systematic review of critically low methodological quality investigating the effect of interventions to improve bone mineral density for people with stroke reported that increased sunlight exposure had no significant effect on falls rate [56].

\section{Models of stroke care interventions}

One systematic review of critically low methodological quality investigating the effect of different models of stroke care on falls outcomes for people with stroke concluded that home rehabilitation with multidisciplinary team outreach had no significant effect on falls rate [56].

\section{GRADE quality of evidence}

The quality of evidence relating to total number of falls, falls rate and number of fallers for each outcome is outlined in the summary of evidence table (see Table 3) and the application of the algorithm to each of these is outlined in Additional file 4.

\section{Discordance between reviews}

Inconsistencies in findings regarding the effectiveness of exercise-based interventions and FES were identified between reviews for people with MS. A potential reason for this difference in findings may be the different selection criteria of the two systematic reviews [50], with Hayes et al. (2019) only including RCTs [51] and Sosnoff and Sung (2015) including both RCTs and NRSIs [52].

Variations in findings regarding the efficacy of exercise-based interventions on falls rate for people with stroke were also identified $[54,56]$, which may be explained by the differences in studies informing outcome, with only one primary study included in both reviews. 


\section{Secondary outcomes}

There were a broad range of secondary outcomes assessed in included systematic reviews including, but not limited to, gait, quality of life, balance, cognition and fatigue. These were assessed with a number of different outcome measures. The effectiveness of falls prevention interventions at improving these outcomes varied across reviews. Of note, very few included reviews provided data regarding adverse events or cost-effectiveness of interventions. The findings regarding secondary outcomes are presented in Additional file 3.

\section{Discussion}

This comprehensive umbrella review included 18 systematic reviews representing 73 unique primary studies investigating the effectiveness of falls prevention interventions at reducing fall events among people with MS, PD and stroke. This review identified low to moderate quality evidence for the effectiveness of exercise-based interventions at reducing falls among people with PD, both over the short-term and long-term. Reviews investigating the effectiveness of exercise-based interventions for people with MS and stroke yielded conflicting results, however, the best available evidence suggests that exercise is effective at reducing falls among people with stroke but no evidence of effect was identified for people with MS. All other types of intervention are relatively under-researched, with insufficient evidence available to draw definitive conclusions regarding their effectiveness.

The large number of systematic reviews that investigated the effectiveness of falls prevention interventions for people with MS, PD and stroke suggest that this field is well researched, particularly among people with PD and stroke. However, this umbrella review has demonstrated that this proliferation in systematic reviews is not reflective of an increase in primary studies but rather that the findings across all of these reviews are largely based on the same small number of trials. The quality and value of a systematic review is largely dependent on the number and methodological quality of included primary studies [69]. Therefore, the inclusion of the same primary studies across many systematic reviews results in the same limitations, such as heterogeneity in methods and outcomes, small sample sizes, and high risk of bias, being reported in newer systematic reviews thus not contributing to advancements in the research field and evidence-based practice. This umbrella review has identified a critical lack of high-quality trials investigating falls prevention interventions for people with MS, PD and stroke, prohibiting researchers and clinicians from drawing firm conclusions regarding their effectiveness.

The finding that exercise is effective at reducing falls among people with PD is consistent with that of Lai et al. (2019) who investigated the effectiveness of exercise-only falls prevention interventions for people with neurological diseases [33]. The inclusion of additional systematic reviews in our umbrella review, along with the application of the GRADE algorithm (showing low to moderate quality evidence), increases the certainty of this finding. However, a difference was noted between the findings of this umbrella review and that of Lai et al. (2019) with respect to the effectiveness of exercise-based interventions for people with stroke. Lai et al. (2019) included two systematic reviews for people with stroke, neither of which found effect. However, two recent systematic reviews identified as part of this umbrella review, neither of which were included in the paper by Lai et al. (2019), found exercise-based interventions significantly improved falls outcomes for people with stroke $[54,68]$. Therefore, the highest quality research and best available evidence for people with stroke, as demonstrated by the AMSTAR 2 and GRADE algorithm, suggests that exercise can reduce falls for these individuals. The evidence regarding the efficacy of exercise-based interventions for people with MS was the least comprehensive, with the smallest amount of primary studies and systematic reviews informing outcomes. The systematic review of the highest methodological quality for people with MS did not find any evidence of effect for exercise-based interventions on falls outcomes but the authors noted that there was an absence of high-quality RCTs in this field meaning that the findings of this review are inconclusive [51]. An umbrella review investigating rehabilitation for people with MS identified high-quality evidence for exercise to improve mobility, muscle strength and fatigue, three common modifiable falls risk factors for people with MS [70]. Consequently, despite the lack of evidence supporting the use of exercise-based interventions for people with MS, a strong theoretical basis for their use remains. There is conclusive evidence regarding the effectiveness of exercise at reducing falls among older adults [71, 72], a much further advanced research field than falls prevention for people with neurological diseases and therefore, further high-quality research is required to determine its effectiveness for people with MS, PD and stroke.

Exercise-based interventions were the most commonly investigated intervention type for all three conditions, supporting the feasibility of a mixed-diagnosis intervention. However, a noticeable difference was identified between the three conditions in terms of the content of these exercise-based interventions. The majority of interventions for people with $\mathrm{PD}$ comprised of mixed training, usually involving some combination of balance, strength and functional or gait exercises. This is in contrast to interventions for people with MS and stroke 
which were primarily focused on one specific training, most commonly balance training or functional movement. Three of the main modifiable falls risk factors for PD, MS and stroke are strength deficits, balance dysfunction and gait impairments and, therefore, it can be hypothesised that the most effective method of reducing falls risk for these individuals would be through a mixed exercise programme, as demonstrated in falls research for older adults [71]. This umbrella review identified low to moderate quality evidence for the efficacy of Tai Chi interventions at improving falls outcomes for people with PD and stroke but this intervention does not have reviews with falls outcomes for people with MS. Another difference was identified between conditions with respect to intervention dosage. A recent review in older adults concluded that interventions consisting of balance training and functional exercises that involved a total weekly dose of three or more hours were most effective at reducing falls rate [71]. Intervention dose was not routinely reported in included reviews but where it was, only four interventions for people with MS and seven for people with stroke achieved this dosage. While formal statistical analyses could not be completed, the findings of this review would suggest that exercise-based interventions that involved mixed training and had a higher intervention dose led to improvements in falls outcomes. Therefore, future trials investigating the efficacy of mixed exercise interventions of sufficient dosage for people with MS, PD and stroke are recommended.

Falls are widely accepted as having a range of causes and, therefore, a surprising finding of this review was the lack of multicomponent or multifactorial interventions that have been investigated for people with MS, PD and stroke. The majority of interventions included in this review were developed with the aim of targeting the physiological falls risk factors, however, psychosocial, environmental and behavioural factors (that are common across these conditions) have also been shown to increase falls risk among individuals with these conditions. Given this broad range of risk factors, it can be hypothesised that multifactorial or multicomponent interventions targeting several risk factors would be more effective at reducing falls. The National Institute for Health and Care Excellence (NICE) guidelines for the assessment and treatment of falls currently recommend the use of multifactorial interventions among older adults [30]. This recommendation is supported by a recent systematic review that found multifactorial interventions reduced falls rate among older adults [73]. The nature of multifactorial interventions, differing components based on individual risk profiles, make them the most likely intervention approach to facilitate groups with mixed neurological conditions. Therefore, research to determine if this multifactorial approach is also effective for people with MS, PD and stroke should be a priority.
There was insufficient evidence identified to make definitive conclusions regarding the effectiveness of technology-based interventions, education-based interventions, environment/assistive technologies, cueing interventions, interventions to improve bone mineral density, and models of stroke care interventions at improving falls outcomes or to compare the interventions across conditions. These interventions were regularly only informed by one primary study. In addition, the quality of evidence for these interventions was primarily low to very low and so further research is warranted to determine their true effectiveness. Given the recent shift to remote treatment delivery, this should be a priority for researchers.

\section{Strengths and limitations}

This umbrella review investigated the effectiveness of non-pharmacological falls prevention interventions for people with MS, PD and stroke and compared the similarities and differences across the three conditions. A robust methodology was used including the development of an a priori protocol, a comprehensive search strategy of databases, grey literature and reference lists, and the use of the AMSTAR 2 and GRADE to determine the strength of the evidence. There were also several limitations to this umbrella review. Firstly, the substantial heterogeneity and overall poor methodological quality of included systematic reviews precludes firm conclusions regarding the effectiveness of these interventions being drawn from the current evidence base. In addition, the language restrictions placed upon our searches may have resulted in relevant citations being missed. However, in an attempt to overcome this, authors of potentially relevant reviews that were published in another language were contacted to determine if an English version was available. Finally, the authors had planned on converting the estimates of effect presented in included reviews to one common measure of effect, however, given that the majority of reviews presented their results narratively, and the variation in interventions and outcomes assessed, this was not possible.

\section{Implications for practice}

Exercise-based interventions are effective at reducing falls among people with PD both over the short- and long-term. The best available evidence identified in this umbrella review suggests that exercise-based interventions can reduce falls for people with stroke but no evidence of effect was identified for people with MS. However, much of the uncertainty regarding the effectiveness of these interventions can be attributed to the lack of high-quality studies informing outcomes and so a strong theoretical rationale remains for the use of exercise-based interventions to address modifiable 
physiological falls risk factors. There was insufficient evidence available for all other intervention types to determine their effectiveness, however, it can be hypothesised that the most effective method of reducing falls risk for individuals with MS, PD and stroke would be through an individualised multifactorial intervention with a core exercise programme and additional elements to address individual, specific needs.

\section{Implications for research}

The authors of every systematic review included in this umbrella review recommended the development of further high-quality primary studies investigating falls prevention interventions for people with MS, PD and stroke, and this recommendation is re-emphasised by the authors of this umbrella review. A key limitation across included systematic reviews was the lack of reporting and heterogeneity of methods of falls data collection, fall definitions and falls outcomes. Particularly of note was the absence of data available across included systematic reviews with respect to injurious falls, with only one review reporting falls outcomes relating to physical injury [54]. Given that a key aim of falls prevention interventions is to reduce injurious falls, this data is of particular interest to service-planners and clinicians when deciding what programme to implement in the community. The large variation across included reviews prohibits cross-comparison of findings and pooling of data. Therefore, there is need for an international standard regarding research methods and outcomes for studies investigating falls among people with PD, MS and stroke.

\section{Conclusions}

Given the negative consequences associated with falls for people with MS, PD and stroke, the development and implementation of theory-based and effective falls prevention interventions is a research priority. Exercisebased interventions have been found to be effective at reducing falls among people with $\mathrm{PD}$, however, the evidence for exercise-based interventions for people with MS and stroke is less conclusive. In addition, conclusions regarding all other intervention types could not be drawn due to insufficient evidence. To progress research in this field, the focus should be on the development of high-quality trials investigating the effectiveness of falls prevention interventions for people with MS, PD and stroke, rather than the publication of further systematic reviews.

\section{Appendix}

Search strategy for CINAHL

S1: TI (falls OR fall* OR "accidental fall") OR AB (falls OR fall* OR "accidental fall").
S2: TI (stroke OR CVA OR cerebrovascular OR apoplexy OR vascular OR MS OR "multiple sclerosis" OR demyelin" OR PD OR "parkinson's disease" OR "parkinson disease" OR parkinson* OR neurol") OR AB (stroke OR CVA OR cerebrovascular OR apoplexy OR vascular OR MS OR "multiple sclerosis" OR demyelin* OR PD OR "parkinson's disease" OR "parkinson disease" OR parkinson* OR neurol*).

S3: (TI stroke OR CVA OR cerebrovascular OR apoplexy OR vascular OR MS OR "multiple sclerosis" OR demyelin" OR PD OR "parkinson's disease" OR "parkinson disease" OR parkinson* OR neurol" OR AB stroke OR CVA OR cerebrovascular OR apoplexy OR vascular OR MS OR "multiple sclerosis" OR demyelin* OR PD OR "parkinson's disease" OR "parkinson disease" OR parkinson* OR neurol*) AND (S1 AND S2).

S4: TI (intervention OR prevention OR rehabilitation OR treatment OR therap*) OR AB (intervention OR prevention OR rehabilitation OR treatment OR therap*).

S5: (TI intervention OR prevention OR rehabilitation OR treatment OR therap* OR AB intervention OR prevention OR rehabilitation OR treatment OR therap*) AND (S3 AND S4).

S6: TI (systematic OR review OR "meta-analysis") OR $\mathrm{AB}$ (systematic OR review OR "meta-analysis").

S7: (TI systematic OR review OR "meta-analysis" OR AB systematic OR review OR "meta-analysis") AND (S5 AND S6)

\section{Abbreviations}

PD: Parkinson's Disease; MS: Multiple Sclerosis; RCT: Randomised Controlled Trial; PRIOR: Preferred Reporting Items for Overviews of Reviews; JBI: Joanna Briggs Institute; PRISMA: Preferred Reporting Items for Systematic Reviews and Meta-Analysis; AMSTAR 2: A MeaSurement Tool to Assess Systematic Reviews 2; GRADE: Grading of Recommendations Assessments, Development and Evaluation; FES: Functional Electrical Stimulation; NICE: National Institute for Health and Care Excellence

\section{Supplementary Information}

The online version contains supplementary material available at https://doi. org/10.1186/s12883-021-02402-6.

Additional file 1. Reasons for exclusions following full-text screen.

Additional file 2. Matrix of evidence table demonstrating degree of overlap and citation count of systematic reviews meeting inclusion criteria.

Additional file 3. Characteristics of included reviews.

Additional file 4. Application of GRADE algorithm to falls outcomes.

\section{Acknowledgements}

This work was supported by the Irish Research Council through the Government of Ireland Postgraduate Scholarship Programme under award number GOIPG/2018/3379 and by the Health Research Board (HRB) of Ireland through the HRB Collaborative Doctoral Awards under Grant CDA2018-005 ("Right Care" Programme).

Authors' contributions

NO'M contributed to the original idea, study design, data acquisition, data analysis, interpretation of data, and drafting and revising of the manuscript. 
AC contributed to the original idea, study design, interpretation of the data, critical revision of the manuscript and supervision of the study. MC contributed to the study design, data analysis and interpretation of data. BC contributed to the data acquisition, data analysis and revision of the manuscript. SC contributed to the original idea, study design, interpretation of the data, critical revision of the manuscript and supervision of the study. All authors read and approved the final manuscript.

\section{Funding}

NO'M is a postgraduate scholar funded by the Irish Research Council through the Government of Ireland Postgraduate Scholarship Programme under award number GOIPG/2018/3379. MC is a postgraduate scholar funded by the Health Research Board (HRB) of Ireland through the HRB Collaborative Doctoral Awards under Grant CDA-2018-005 ("Right Care" Programme). The funders had no role in study design, data collection and analysis, decision to publish, or preparation of the manuscript.

\section{Availability of data and materials}

All data generated or analysed during this study are included in this published article and its supplementary information files.

\section{Declarations}

\section{Ethics approval and consent to participate}

Not applicable.

\section{Consent for publication}

Not applicable.

\section{Competing interests}

$\mathrm{SC}$ is contracted to deliver a patient support programme for Novartis. All other authors declare that they have no competing interests.

\section{Author details}

${ }^{1}$ School of Allied Health, Faculty of Education and Health Sciences, University of Limerick, Limerick, Ireland. ${ }^{2}$ Ageing Research Centre, Health Research Institute, University of Limerick, Limerick, Ireland. ${ }^{3}$ Department of Physical Education and Sport Sciences, Faculty of Education and Health Sciences, University of Limerick, Limerick, Ireland. ${ }^{4}$ Centre of Physical Activity for Health, Health Research Institute, University of Limerick, Limerick, Ireland.

${ }^{5}$ Multiple Sclerosis Society of Ireland, Limerick, Ireland.

Received: 19 April 2021 Accepted: 29 August 2021

\section{Published online: 29 September 2021}

\section{References}

1. Sackley C, Brittle N, Patel S, Ellins J, Scott M, Wright C, et al. The prevalence of joint contractures, pressure sores, painful shoulder, other pain, falls, and depression in the year after a severely disabling stroke. Stroke. 2008;39(12): 3329-34. https://doi.org/10.1161/STROKEAHA.108.518563.

2. Nilsagard Y, Gunn H, Freeman J, Hoang P, Lord S, Mazumder R, et al. Falls in people with MS--an individual data meta-analysis from studies from Australia, Sweden, United Kingdom and the United States. Mult Scler. 2015; 21(1):92-100. https://doi.org/10.1177/1352458514538884.

3. Paul SS, Canning CG, Sherrington C, Lord SR, Close JC, Fung VS. Three simple clinical tests to accurately predict falls in people with Parkinson's disease. Mov Disord. 2013;28(5):655-62. https://doi.org/10.1002/mds.25404.

4. Beghi E, Gervasoni E, Pupillo E, Bianchi E, Montesano A, Aprile I, et al. Prediction of falls in subjects suffering from Parkinson disease, multiple sclerosis, and stroke. Arch Phys Med Rehabil. 2018;99(4):641-51. https://doi. org/10.1016/j.apmr.2017.10.009.

5. Gunn H, Creanor S, Haas B, Marsden J, Freeman J. Frequency, characteristics, and consequences of falls in multiple sclerosis: findings from a cohort study. Arch Phys Med Rehabil. 2014;95(3):538-45. https://doi.org/10.1016/j.apmr.2 013.08.244.

6. Gazibara T, Kisic-Tepavcevic D, Svetel M, Tomic A, Stankovic I, Kostic VS, et al. Indoor and outdoor falls in persons with Parkinson's disease after 1 year follow-up study: differences and consequences. Neurol Sci. 2016;37(4): 597-602. https://doi.org/10.1007/s10072-016-2504-2.

7. Schmid AA, Yaggi HK, Burrus N, McClain V, Austin C, Ferguson J, et al. Circumstances and consequences of falls among people with chronic stroke. J Rehabil Res Dev. 2013;50(9):1277-86. https://doi.org/10.1682/JRRD.2 012.11.0215.

8. Comber L, Coote S, Finlayson M, Galvin R, Quinn G, Peterson E. An exploration of fall-related, psychosocial variables in people with multiple sclerosis who have fallen. Br J Occup Ther. 2017;80(10):587-95. https://doi. org/10.1177/0308022617725492.

9. Schmid AA, Rittman M. Consequences of Poststroke falls: activity limitation, increased dependence, and the development of fear of falling. Am J Occup Ther. 2009;63(3):310-6. https://doi.org/10.5014/ajot.63.3.310.

10. Brožová $H$, Štochl J, Roth J, Růžička E. Fear of falling has greater influence than other aspects of gait disorders on quality of life in patients with Parkinson's disease. Neuroendocrinol Lett. 2009;30(4):101-5.

11. Critchley RJ, Khan SK, Yarnall AJ, Parker MJ, Deehan DJ. Occurrence, management and outcomes of hip fractures in patients with Parkinson's disease. Br Med Bull. 2015;115(1):135-42. https://doi.org/10.1093/bmb/ldv02 9.

12. (HSE) HSE. National strategy \& policy for the provision of neurorehabilitation services in Ireland: Implementation framework 2019-2021. https://www.hse.ie/eng/services/list/4/disability/neurorehabilitation/nationalstrategy-policy-for-the-provision-of-neuro-rehabilitation-services-in-ireland. pdf (2019). Accessed 11/03/2020.

13. Gunn H, Endacott R, Haas B, Marsden J, Freeman J. Development of a balance, safe mobility and falls management programme for people with multiple sclerosis. Disabil Rehabil. 2018;40(24):2857-66. https://doi.org/10.1 080/09638288.2017.1362041

14. Kersten P, Ellis-Hill C, McPherson KM, Harrington R. Beyond the RCT understanding the relationship between interventions, individuals and outcome - the example of neurological rehabilitation. Disabil Rehabil. 2010; 32(12):1028-34. https://doi.org/10.3109/09638281003775568.

15. Deb P, Sharma S, Hassan KM. Pathophysiologic mechanisms of acute ischemic stroke: an overview with emphasis on therapeutic significance beyond thrombolysis. Pathophysiology. 2010;17(3):197-218. https://doi.org/1 0.1016/j.pathophys.2009.12.001.

16. Ghasemi N, Razavi S, Nikzad E. Multiple sclerosis: pathogenesis, symptoms, diagnoses and cell-based therapy. Cell J. 2017;19(1):1-10. https://doi.org/1 0.22074/cellj.2016.4867.

17. Kouli A, Torsney KM, Kuan WL. Parkinson's disease: etiology, Neuropahtology, and pathogenesis. In: Stoker TB, Greenland JC, editors. Parkinson's disease: pathogenesis and clinical aspects. Brisbabe, Australia: Codon Publications; 2018. https://doi.org/10.15586/codonpublications.parkinsonsdisease.2018.ch1.

18. Woodruff TM, Thundyil J, Tang SC, Sobey CG, Taylor SM, Arumugam TV. Pathophysiology, treatment, and animal and cellular models of human ischemic stroke. Mol Neurodegener. 2011;6(1):11. https://doi.org/10.1186/1 750-1326-6-11.

19. Xu T, Clemson L, O'Loughlin K, Lannin NA, Dean C, Koh G. Risk factors for falls in community stroke survivors: a systematic review and Meta-analysis. Arch Phys Med Rehabil. 2018;99(3):563-73. https://doi.org/10.1016/j.apmr.2 017.06.032.

20. Pang MY, Eng JJ. Fall-related self-efficacy, not balance and mobility performance, is related to accidental falls in chronic stroke survivors with low bone mineral density. Osteoporos Int. 2008;19(7):919-27. https://doi. org/10.1007/s00198-007-0519-5.

21. Goto Y, Otaka Y, Suzuki K, Inoue S, Kondo K, Shimizu E. Incidence and circumstances of falls among community-dwelling ambulatory stroke survivors: a prospective study. Geriatr Gerontol Int. 2019;19(3):240-4. https:// doi.org/10.1111/ggi.13594.

22. Costa AGdS, Oliveira-Kumakura ARdS, Araujo TLd, Castro NBd, Silva VMd, Lopes MVdO. Stroke and risk factors for falls in elderly individuals. Revista da Rede de Enfermagem do Nordeste. 2017;18 5; https://doi.org/10.15253/21 75-6783.2017000500014.

23. Gunn HJ, Newell P, Haas B, Marsden JF, Freeman JA. Identification of risk factors for falls in multiple sclerosis: a systematic review and Meta-analysis. Phys Ther. 2013;93(4):504-13. https://doi.org/10.2522/ptj.20120231.

24. Kasser SL, Jacobs JV, Foley JT, Cardinal BJ, Maddalozzo GF. A prospective evaluation of balance, gait, and strength to predict falling in women with multiple sclerosis. Arch Phys Med Rehabil. 2011;92(11):1840-6. https://doi. org/10.1016/j.apmr.2011.06.004.

25. Hoang PD, Cameron MH, Gandevia SC, Lord SR. Neuropsychological, balance, and mobility risk factors for falls in people with multiple sclerosis: a prospective cohort study. Arch Phys Med Rehabil. 2014;95(3):480-6. https:// doi.org/10.1016/j.apmr.2013.09.017. 
26. Carling A, Forsberg A, Nilsagard Y. Falls in people with multiple sclerosis: experiences of 115 fall situations. Clin Rehabil. 2018;32(4):526-35. https://doi. org/10.1177/0269215517730597.

27. Canning CG, Paul SS, Nieuwboer A. Prevention of falls in Parkinson's disease: a review of fall risk factors and the role of physical interventions. Neurodegen Dis Manage. 2014;4(3):203-21. https://doi.org/10.2217/ nmt.14.22.

28. Pelicioni PHS, Menant JC, Latt MD, Lord SR. Falls in Parkinson's disease subtypes: risk factors, Locations and Circumstances. Int J Environ Res Public Health. 2019;16(12):12. https://doi.org/10.3390/ijerph16122216.

29. Ge HL, Chen XY, Lin YX, Ge TJ, Yu LH, Lin ZY, et al. The prevalence of freezing of gait in Parkinson's disease and in patients with different disease durations and severities. Chin Neurosurg J. 2020;6(1):17. https://doi.org/10.11 86/s41016-020-00197-y.

30. NICE. Assessment and prevention of falls in older people. www.nice.org.uk guidance/CG161 (2013). Accessed.

31. Panel on Prevention of Falls in Older Persons AGS, British Geriatrics S. Summary of the Updated American Geriatrics Society/British Geriatrics Society clinical practice guideline for prevention of falls in older persons. J Am Geriatr Soc. 2011;59 1:148-57; https://doi.org/10.1111/j.1532-5415.2010. 03234.x.

32. Cattaneo D, Gervasoni E, Pupillo E, Bianchi E, Aprile I, Imbimbo I, et al. Educational and exercise intervention to prevent falls and improve participation in subjects with neurological conditions: the NEUROFALL randomized controlled trial. Front Neurol. 2019;10:865. https://doi.org/10.33 89/fneur.2019.00865.

33. Lai CH, Chen HC, Liou TH, Li W, Chen SC. Exercise Interventions for Individuals with Neurological Disorders: A Systematic Review of Systematic Reviews. Am J Phys Med Rehabil. 2019;98(10):921-30. https://doi.org/10.1 097/PHM.0000000000001247.

34. Aromataris E, Fernandez R, Godfrey C, Holly C, Khalil H, Tungpunkom P. Chapter 10: umbrella reviews. In: Aromataris E, Munn Z (editors). Joanna Briggs institute Reviewer's manual. https://reviewersmanual.joannabriggs. org/ (2017). Accessed.

35. Liberati A, Altman DG, Tetzlaff J, Mulrow C, Gotzsche PC, loannidis JP, et al. The PRISMA statement for reporting systematic reviews and meta-analyses of studies that evaluate healthcare interventions: explanation and elaboration. BMJ. 2009:339(jul21 1):b2700. https://doi.org/10.1136/bmj.b2700.

36. Moher D, Liberati A, Tetzlaff J, Altman DG, Group P. Preferred reporting items for systematic reviews and meta-analyses: the PRISMA statement. BMJ. 2009;339(jul21 1):b2535. https://doi.org/10.1136/bmj.b2535.

37. Pollock M, Fernandes RM, Pieper D, Tricco AC, Gates M, Gates A, et al. Preferred Reporting Items for Overviews of Reviews (PRIOR): a protocol for development of a reporting guideline for overviews of reviews of healthcare interventions. Syst Rev. 2019;8(1):335. https://doi.org/10.1186/s13 643-019-1252-9.

38. Hunt $H$, Pollock A, Campbell $P$, Estcourt L, Brunton G. An introduction to overviews of reviews: planning a relevant research question and objective for an overview. Syst Rev. 2018;7(1):-39. https://doi.org/10.1186/s13643-0180695-8.

39. O'Malley N, Clifford AM, Comber L, Coote S. Effectiveness of nonpharmacological falls prevention interventions for people with Multiple Sclerosis, Parkinson's Disease and stroke: protocol for an umbrella review. HRB Open Res. 2020;3. https://doi.org/10.12688/hrbopenres.13023.1.

40. Aromataris E, Fernandez R, Godfrey CM, Holly C, Khalil H, Tungpunkom P. Summarizing systematic reviews: methodological development, conduct and reporting of an umbrella review approach. Int J Evid Based Healthc. 2015;13(3):132-40. https://doi.org/10.1097/XEB.0000000000000055.

41. Garner P, Hopewell S, Chandler J, MacLehose H, Schunemann HJ, Akl EA, et al. When and how to update systematic reviews: consensus and checklist. BMJ. 2016;354:i3507. https://doi.org/10.1136/bmj.i3507.

42. O'Malley N, Clifford AM, Comber L, Coote S. Fall definitions, faller classifications and outcomes used in falls research among people with multiple sclerosis: a systematic review. Disabil Rehabil. 2020:1-8. https://doi. org/10.1080/09638288.2020.1786173.

43. Hines $H$, Kynoch $K$, Khalil $H$. Effectiveness of interventions to prevent medication errors: an umbrella review systematic review protocol. JBI Database of Systematic Reviews \& Implementation Reports. 2018;16 2:291-6; https://doi.org/10.11124/JBISRIR-2017-003481.

44. Husser T, Marcom J, Mark J, Buonora J, Benham B. Effectiveness of nonopioid pharmacological adjuncts for adult surgical patients: an umbrella review protocol. JBI Database of Systematic Reviews \& Implementation Reports. 2019;17 7:1319-25; https://doi.org/10.11124/JBISRIR-2017-003960.

45. McKenzie JE, Brennan SE. Overviews of systematic reviews: great promise, greater challenge. Syst Rev. 2017;6 1:185; https://doi.org/10.1186/s13643-01 7-0582-8.

46. Pollock M, Fernandes RM, Newton AS, Scott SD, Hartling L. A decision tool to help researchers make decisions about including systematic reviews in overviews of reviews of healthcare interventions. Syst Rev. 2019;8 1:29; https://doi.org/10.1186/s13643-018-0768-8.

47. Shea BJ, Reeves BC, Wells G, Thuku M, Hamel C, Moran J, et al. AMSTAR 2: a critical appraisal tool for systematic reviews that include randomised or non-randomised studies of healthcare interventions, or both. BMJ. 2017;358: j4008. https://doi.org/10.1136/bmj.j4008.

48. Pieper D, Buechter $R$, Jerinic $P$, Eikermann M. Overviews of reviews often have limited rigor: a systematic review. J Clin Epidemiol. 2012;65(12):126773. https://doi.org/10.1016/j.jclinepi.2012.06.015.

49. Pollock A, Farmer SE, Brady MC, Langhorne P, Mead GE, Mehrholz J, et al. An algorithm was developed to assign GRADE levels of evidence to comparisons within systematic reviews. J Clin Epidemiol. 2016;70:106-10. https://doi.org/10.1016/j.jclinepi.2015.08.013.

50. Jadad AR, Cook DJ, Browman GP. A guide to interpreting discordant systematic reviews. Can Med Assoc. 1997;156:1411-6.

51. Hayes S, Galvin R, Kennedy C, Finlayson M, McGuigan C, Walsh CD, et al. Interventions for preventing falls in people with multiple sclerosis. The Cochrane database of systematic reviews. 2019;11:CD012475. https://doi. org/10.1002/14651858.CD012475.pub2.

52. Sosnoff JJ, Sung J. Reducing falls and improving mobility in multiple sclerosis. Expert Rev Neurother. 2015;15(6):655-66. https://doi.org/10.1 586/14737175.2015.1046377.

53. Booth V, Masud T, Connell L, Bath-Hextall F. The effectiveness of virtual reality interventions in improving balance in adults with impaired balance compared with standard or no treatment: a systematic review and metaanalysis. Clin Rehabil. 2014;28(5):419-31. https://doi.org/10.1177/0269215513 509389.

54. Denissen S, Staring W, Kunkel D, Pickering RM, Lennon S, Geurts AC, et al. Interventions for preventing falls in people after stroke. Cochrane Database Syst Rev. 2019;10:CD008728. https://doi.org/10.1002/14651858.CD008728.pub3.

55. Pollock A, Gray C, Culham E, Durward BR, Langhorne P. Interventions for improving sit-to-stand ability following stroke. Cochrane Database Syst Rev. 2014; 5:Cd007232; https://doi.org/10.1002/14651858.CD007232.pub4.

56. Batchelor F, Hill K, Mackintosh S, Said C. What works in falls prevention after stroke? A systematic review and meta-analysis. Stroke. 2010;41(8):1715-22. https://doi.org/10.1161/STROKEAHA.109.570390.

57. Rutz DG, Benninger DH. Physical therapy for freezing of gait and gait impairments in Parkinson's disease: a systematic review. PM \& R : J Injury, Func, Rehab. 2020;12(11):1140-56. https://doi.org/10.1002/pmrj.12337.

58. Owen $\mathrm{CL}$, Ibrahim K, Dennison L, Roberts HC. Falls self-management interventions for people with Parkinson's disease: a systematic review. J Parkinson's Dis. 2019:9(2):283-99.

59. Rodrigues-Krause J, Krause M, Reischak-Oliveira A. Dancing for healthy aging: functional and metabolic perspectives. Altern Ther Health Med. 2019; 25(1):44-63.

60. Winser SJ, Paul LF, Magnus LKL, Yan S, Shenug TP, Sing YM, et al. Economic evaluation of exercise-based fall prevention programs for people with Parkinson's disease: a systematic review. J Altern Complem Med. 2019; 25(12):1225-37. https://doi.org/10.1089/acm.2019.0148.

61. Mak MK, Wong-Yu IS, Shen X, Chung CL. Long-term effects of exercise and physical therapy in people with Parkinson disease. Nat Rev Neurol. 2017;13(11):689-703. https://doi.org/10.1038/nrneurol.2 017.128 .

62. Ramazzina I, Bernazzoli B, Costantino C. Systematic review on strength training in Parkinson's disease: an unsolved question. Clin Interv Aging. 2017;12:619-28. https://doi.org/10.2147/cia.S131903.

63. Song R, Grabowska W, Park M, Osypiuk K, Vergara-Diaz GP, Bonato P, et al. The impact of tai chi and Qigong mind-body exercises on motor and nonmotor function and quality of life in Parkinson's disease: a systematic review and meta-analysis. Parkinsonism Relat Disord. 2017;41:3-13. https://doi.org/1 0.1016/.j.parkreldis.2017.05.019.

64. Shen X, Wong-Yu IS, Mak MK. Effects of exercise on falls, balance, and gait ability in Parkinson's disease: a Meta-analysis. Neurorehabil Neural Repair. 2016;30(6):512-27. https://doi.org/10.1177/1545968315613447. 
65. Tomlinson CL, Herd CP, Clarke CE, Meek C, Patel S, Stowe R, et al. Physiotherapy for Parkinson's disease: a comparison of techniques. Cochrane Database Syst Rev. 2014;6:Cd002815. https://doi.org/10.1002/14 651858.CD002815.pub2.

66. Tomlinson CL, Patel S, Meek C, Clarke CE, Stowe R, Shah L, et al. Physiotherapy versus placebo or no intervention in Parkinson's disease. Cochrane Database Syst Rev. 2012; 8:Cd002817; https://doi.org/10.1002/14 651858.CD002817.pub3.

67. Monti S, Bellini C, Medri E, Pillastrini P. Physiotherapy and the prevention of falls in Parkinson's disease: scientific evidences in the literature. Scienza Riabilitativa. 2011;13(3):28-35.

68. Winser SJ, Tsang WW, Krishnamurthy K, Kannan P. Does tai chi improve balance and reduce falls incidence in neurological disorders? A systematic review and meta-analysis. Clin Rehabil. 2018;32(9):1157-68. https://doi.org/1 $0.1177 / 0269215518773442$.

69. Moller AM, Myles PS. What makes a good systematic review and metaanalysis? Br J Anaesth. 2016;1 17(4):428-30. https://doi.org/10.1093/bja/aew264.

70. Khan F, Amatya B. Rehabilitation in multiple sclerosis: a systematic review of systematic reviews. Arch Phys Med Rehabil. 2017;98(2):353-67. https://doi. org/10.1016/j.apmr.2016.04.016.

71. Sherrington C, Fairhall N, Kwok W, Wallbank G, Tiedemann A, Michaleff ZA, et al. Evidence on physical activity and falls prevention for people aged $65+$ years: systematic review to inform the WHO guidelines on physical activity and sedentary behaviour. Int J Behav Nutr Phys Act. 2020;17(1):144. https://doi.org/10.1186/s12966-020-01041-3.

72. Sherrington C, Fairhall N, Wallbank G, Tiedemann A, Michaleff ZA, Howard K, et al. Exercise for preventing falls in older people living in the community: an abridged Cochrane systematic review. Br J Sports Med. 2020;54(15):88591. https://doi.org/10.1136/bjsports-2019-101512.

73. Hopewell S, Copsey B, Nicolson P, Adedire B, Boniface G, Lamb S. Multifactorial interventions for preventing falls in older people living in the community: a systematic review and meta-analysis of 41 trials and almost 20000 participants. Br J Sports Med. 2020;54(22):1340-50. https://doi.org/1 0.1136/bjsports-2019-100732.

\section{Publisher's Note}

Springer Nature remains neutral with regard to jurisdictional claims in published maps and institutional affiliations.

\section{Ready to submit your research? Choose BMC and benefit from:}

- fast, convenient online submission

- thorough peer review by experienced researchers in your field

- rapid publication on acceptance

- support for research data, including large and complex data types

- gold Open Access which fosters wider collaboration and increased citations

- maximum visibility for your research: over $100 \mathrm{M}$ website views per year

At $\mathrm{BMC}$, research is always in progress.

Learn more biomedcentral.com/submissions 